DIVISION OF THE HUMANITIES AND SOCIAL SCIENCES

CALIFORNIA INSTITUTE OF TECHNOLOGY

PASADENA, CALIFORNIA 91125

BUDGET BALANCEDNESS AND OPTIMAL INCOME TAXATION

Marcus Berliant*

California Institute of Technology and

Washington University

Frank H. Page, Jr.

University of Alabama

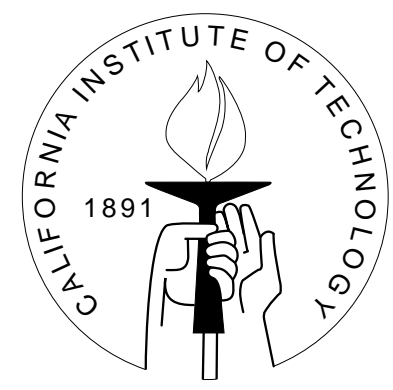

SOCIAL SCIENCE WORKING PAPER 1164

May 2003 


\title{
Budget Balancedness and Optimal Income Taxation
}

\author{
Marcus Berliant* Frank H. Page, Jr.
}

\begin{abstract}
We make two main contributions to the theory of optimal income taxation. First, assuming conditions sufficient for existence of a Pareto optimal income tax and public goods mechanism, we show that if, in addition, agents' preferences satisfy an extended notion of single crossing called capacity constrained single crossing, then there exists a Pareto optimal income tax and public goods mechanism that is budget balancing. Second, we show that, even without capacity constrained single crossing, existence of a budget balancing, Pareto optimal income tax and public goods mechanism is guaranteed if the set of agent types contains no atoms.
\end{abstract}

Keywords: Optimal income taxation; Budget balancedness; Mechanism design; Implementation

JEL classification numbers: $\mathrm{D} 8, \mathrm{C} 7, \mathrm{H} 2$

*The current version of this paper was completed while the first author was visiting Caltech. An earlier version of this paper, entitled "Income Taxes and the Provision of Public Goods: Optima and Balanced Government Budgets," was completed while the second author was visiting CERMSEM, Paris I (Pantheon-Sorbonne). Both authors thank these institutions for support and hospitality. Both authors also thank seminar participants at UC Riverside and Vanderbilt University for many helpful comments. Berliant gratefully acknowledges financial support from the National Science Foundation, grant number SBR 93 19994, and the American Philosophical Society. Page gratefully acknowledges financial support from the University of Alabama and CERMSEM. The authors bear full responsibility for any remaining errors. Email addresses: Marcus Berliant (berliant@wueconc.wustl.edu); Frank H. Page, Jr. (fpage@cba.ua.edu) 


\section{Introduction}

Beginning with the work of Vickrey (1945) and Mirrlees (1971), economists have used models of optimal income taxation for policy prescriptions and for the normative analysis of government behavior. By now, a great deal of progress has been made on the issues of existence and characterization of optimal income taxes. ${ }^{1}$ A third issue, budget balancedness, has received much less attention. The purpose of this paper is to address this issue within the context of a model in which both income taxes and public goods are chosen endogenously. We make two contributions to the theory budget balancedness and optimal income taxation. First, assuming conditions sufficient for existence of a Pareto optimal income tax and public goods mechanism, we show that if agents' preferences satisfy an extended notion of single crossing we call capacity constrained single crossing (i.e., a condition implied by the classical SpenceMirrlees condition), then existence of a Pareto optimal income tax and public goods mechanism that is budget balancing is guaranteed. Second, we show that existence of a budget balancing, Pareto optimal income tax and public goods mechanism is also guaranteed if capacity constrained single crossing is replaced by the assumption that set of agent types contains no atoms. The atomless condition will be satisfied automatically if, for example, the distribution of agent types is given by a continuous density function.

What is intriguing about the problem of budget balancedness is that despite the fact that agent preferences are strictly decreasing in income taxes, a budget surplus can occur even under a second best Pareto optimal income tax and public goods mechanism. Moreover, any attempt to spend the surplus by increasing public goods levels may in fact exacerbate the problem - because such increases may alter agents' marginal rates of substitution (income for tax liability) in ways that induce agents to choose even higher levels of income and tax liability (e.g., public goods and income may be complementary "goods"). ${ }^{2}$ Thus, the anomaly of the government burning money to maintain optimality can occur.

The existence of a Pareto optimal and budget balancing income tax and public goods mechanism depends critically upon the shape of the tax and income possibilities set (i.e., the set of all possible aggregate income and tax revenue pairs attainable under some implementable income tax and public goods mechanism). Here, we introduce the notion of tax convexity. We say that the tax and income possibilities set is tax convex if any convex combination of attainable aggregate tax revenue is also attainable. Our two main contributions to the theory of budget balancedness and optimal income taxation are consequences of three fundamental results related to

\footnotetext{
${ }^{1}$ See Myles (1995) for a survey. Also, see Berliant and Page (2001) for results on existence.

${ }^{2}$ Note, however, that if agent utility functions are additively separable in public goods, then agents' marginal rates of substitution (income for tax liability) will not be altered by increasing public goods quantities. In our model, we allow for more general trade-offs between income and tax liability. Since the focus of our model is on this trade-off, we do not assume that agent utility functions are additively separable.
} 
tax convexity. First, we show that if the tax and income possibilities set is tax convex, then there exists an optimal income tax-public goods mechanism that is budget balancing. Second, we show that if the capacity constrained single crossing condition is satisfied, then the tax and income possibilities set is tax convex. Third, we show that if the atomless condition is satisfied, then again the tax and income possibilities set is tax convex.

As in Berliant and Page (2001), here we proceed by transforming the income taxpublic goods mechanism design problem to an equivalent problem of optimal menu design (e.g., see Hammond (1979), Holmstrom (1984) and Page (1992)). This transformation is the key ingredient which allows us to establish the connection between tax convexity and budget balancedness, the connection between single crossing and tax convexity, and finally the connection between the atomless condition and tax convexity. The validity of this transformation rests upon another fundamental result - a result characterizing all implementable income tax-public goods mechanisms in terms of revenue feasible menu and public goods pairs. Here, we shall state (and prove in the appendix) this characterization result, and for the sake of the reader, we shall restate our result on the equivalence of the income tax-public goods mechanism design problem and the menu design problem (i.e., Theorem 2 in Berliant and Page (2001)).

In analyzing the problem of budget balancedness, we allow both the income tax and the public goods levels to be chosen endogenously. This differentiates our model from the classical model of optimal income taxation where public goods levels are fixed (e.g., see Mirrlees (1971)). Moreover, while we allow public goods levels to vary, we treat only the pure public goods case and focus on nonlinear income taxation. This differentiates our model from recent models of taxation and the provision of local public goods (see, for example, Conley and Wooders (2001)). We view our work here as providing a foundation for future investigations of the role played by budget balancedness in multi-jurisdictional competition via income taxes and local public goods. Our work on nonlinear income taxation also contrasts with the literature on linear commodity taxation, as represented for example by Diamond and Mirrlees (1971). In a more recent paper in this literature, Konishi (1995) has analyzed the problem of Pareto-improving linear commodity taxation under an exogenously given nonlinear income tax.

The model we develop, while similar to models found in the principal-agent literature (e.g., Mirrlees (1976), Holmstrom (1979)), differs from the classical principalagent model in several important respects. First, rather than having a single agent, there are many agents, the taxpayers. Second, in our model once the agent has chosen an action, he faces no uncertainty. Specifically, in our model each agent chooses a level of income rather than, as is the case in the principal-agent model, a probability distribution over income. Third, while the principal-agent literature often restricts to linear or quasi-linear objective or utility functions, the focus of the optimal income tax model is on the trade-off between labor/leisure and consumption (i.e., the 
trade-off between income and tax liability), so we allow more general preferences. Finally, in our model there are no voluntary participation (or individual rationality) constraints. These constraints are replaced by a financing constraint which requires that the government choose an income tax mechanism that funds the public goods. Thus, formally our model and the principal-agent model are quite different.

In Section 2, we present the model. In Section 3, we present our main results and we discuss the intuition behind our results. In Sections 4-6, we present our formal analysis of budget balancedness and optimal income taxation. Thus, Section 4-6 are technical in nature. All proofs are gathered in Section 6. 


\section{The Model}

\subsection{Basic Ingredients}

We begin by specifying the set $K$ of all feasible (non-bankrupting) income and tax liability pairs. Let $\mathbb{Y}:=[0, m]$ and $\mathbb{T}:=[s, m]$ for some numbers $m>0$ and $s \leq 0$. Define $K$ as follows:

$$
K:=\{(y, \tau) \in \mathbb{Y} \times \mathbb{T}: y \geq \tau\} .
$$

For any pair $(y, \tau) \in \mathbb{Y} \times \mathbb{T}, y$ denotes income and $\tau$ denotes the corresponding tax liability. Note that if $s<0$, then tax liabilities can be negative, indicating transfer payments. Equipped with the standard Euclidean metric, $d_{e}, K$ is compact.

Let $W$ denote the set of agent types, usually called ability or wage rates in the literature, and equip $W$ with a $\sigma$-field $\Sigma$ and a finite measure $\eta(\cdot)$ defined on $\Sigma$. For $E \in \Sigma, \eta(E)$ is the number of agents of type $w \in E$.

In the standard optimal income tax model, the upper bound on labor supply (hours worked) in combination with the wage rate imply an individual-specific, exogenous upper bound on the income an individual can earn. This constraint, which we call the capacity constraint, is rarely made explicit in the literature. The reason for this is that most of the literature focuses on necessary conditions for optimal income taxes rather than budget balancedness. Here we must make this constraint formal. To begin, for each $w \in W$ let $m(w)$ denote the maximum earned income level attainable by a type $w$ agent. We will assume that the function $m(\cdot)$ satisfies the following assumptions:

$[\mathrm{A}-1]$

(1) For all $w \in W, 0<m(w) \leq m$.

(2) The function $m(\cdot)$ is $(\Sigma, B((0, m]))$-measurable. ${ }^{3}$

Define the set $Y(w)$ as follows:

$$
Y(w):=\{y \in \mathbb{Y}: 0 \leq y \leq m(w)\} .
$$

The interval $Y(w)$ is the subset of income levels in $\mathbb{Y}:=[0, m]$ attainable by an agent of type $w$. We will refer to the interval-valued mapping, $w \rightarrow Y(w)$, as the income correspondence. Note that the income correspondence has closed convex values with $0 \in Y(w)$ for all $w \in W$ and that by [A-1](1) (i.e., $0<m(w)$ for all $w \in W$ ) the interior of the income interval $Y(w)$ is nonempty for all $w \in W$.

Example 1 (Agent Types and Maximum Attainable Income): Suppose the set of tax liabilities is given by an interval $\mathbb{T}=[0, m]$ and the set of agent types is given

\footnotetext{
${ }^{3} B((0, m])$ denotes the Borel $\sigma$-field of the interval $(0, m]$. The function $m(\cdot)$ is $(\Sigma, B((0, m])$ measurable iff$$
\{w \in W: m(w) \in E\} \in \Sigma
$$

for $E \in B((0, m])$.
} 
by an interval of positive ability parameters $W=[a, b] \subset \mathbb{R}_{++}$. For each income and tax liability pair $(y, \tau) \in K$, let labor be given by $l=\frac{u}{w}$, and consumption by $c=y-\tau{ }^{4}$ Letting $L>0$ denote the maximum amount of labor that can be supplied by any agent type, we have $l \in[0, L]$ and $y \in[0, w L]$. Thus $m(w):=w L$ and $Y(w):=[0, w L]$.

Suppose now that there are $k$ public goods $z:=\left(z_{1}, \ldots, z_{k}\right) \in \mathbb{R}_{+}^{k}$. We shall assume that the set of all possible public goods vectors is given by the nonnegative orthant $\mathbb{R}_{+}^{k}$ and that for each vector of public goods $z \in \mathbb{R}_{+}^{k}, h(z)$ is the (nonnegative) cost of providing public goods $z$. Also, we shall assume that

$[\mathrm{A}-2]$

(1) The public goods cost function $h(\cdot): \mathbb{R}_{+}^{k} \rightarrow \mathbb{R}_{+}$ is lower semicontinuous. ${ }^{5}$

(2) There exists $M \in \mathbb{R}$ such that for all $z \in \mathbb{R}_{+}^{k}$ with $\|z\|>M$, $h(z)>m \cdot \eta(W)$.

Recall that $m \cdot \eta(W)$ bounds from above the aggregate amount of income that can be generated by all agents. By $[\mathrm{A}-2](2)$, the cost $h(z)$ of producing public goods $z \in \mathbb{R}_{+}^{k}$ of magnitude $\|z\|>M$ exceeds this bound on aggregate income. Thus, under assumption [A-2] (2) for public goods $z$ of magnitude $\|z\|>M$, even if income $m \cdot \eta(W)$ were transferred to the government as tax revenue, it would be insufficient to finance public goods $z$.

\section{2 income tax-public goods Mechanisms}

An income tax-public goods mechanism is a 3 -tuple $(y(\cdot), t(\cdot), z)$ where $y(\cdot): W \rightarrow \mathbb{Y}$ is a direct mechanism mapping from agent types into income, $t(\cdot): \mathbb{Y} \rightarrow \mathbb{T}$ is an indirect mechanism mapping from income into tax liabilities, and $z \in \mathbb{R}_{+}^{k}$ is a vector of public goods. The quantity $y(w)$ is the income level chosen by a type $w$ agent, while $t(y(w))$ is the corresponding tax liability given income tax function $t(\cdot)$.

Let $M(W, \mathbb{Y})$ denote the set of all $(\Sigma, B(\mathbb{Y}))$-measurable functions $y(\cdot): W \rightarrow \mathbb{Y}$, and $M(\mathbb{Y}, \mathbb{T})$ the set of all $(B(\mathbb{Y}), B(\mathbb{T}))$-measurable functions $t(\cdot): \mathbb{Y} \rightarrow \mathbb{T}^{6}$ We

\footnotetext{
${ }^{4}$ Thus gross labor income is given by $y=l \cdot w$.

${ }^{5}$ The function $h(\cdot): \mathbb{R}_{+}^{k} \rightarrow \mathbb{R}_{+}$is lower semicontinuous if $z_{n} \rightarrow z$ implies that

$$
\liminf h\left(z_{n}\right) \geq h(z)
$$

${ }^{6} B(\mathbb{T})$ denotes the Borel $\sigma$-field in $\mathbb{T}$ and $B(\mathbb{Y})$ denotes the Borel $\sigma$-field in $\mathbb{Y}$. A function $y(\cdot): W \rightarrow \mathbb{Y}$ is $(\Sigma, B(\mathbb{Y}))$-measurable iff

$$
\{w \in W: y(w) \in E\} \in \Sigma
$$
}

for $E \in B(\mathbb{Y})$. $(B(\mathbb{Y}), B(\mathbb{T}))$-measurability is defined in a similar manner. 
shall take as the feasible set of direct income functions the set $S(Y(\cdot))$ given by

$$
S(Y(\cdot)):=\{y(\cdot) \in M(W, \mathbb{Y}): y(w) \in Y(w) \text { for all } w \in W\} .
$$

Thus, a direct income function, $y(\cdot): W \rightarrow \mathbb{Y}$, is feasible if and only if it is (i) measurable and (ii) $0 \leq y(w) \leq m(w)$ for all $w \in W$.

For each $y \in \mathbb{Y}$, let

$$
T(y):=\{\tau \in T: s \leq \tau \leq y\} .
$$

We shall take as the feasible set income tax functions the set $S(T(\cdot))$ given by

$$
S(T(\cdot)):=\{t(\cdot) \in M(\mathbb{Y}, \mathbb{T}): t(y) \in T(y) \text { for all } y \in \mathbb{Y}\}
$$

Thus, an income tax function, $t(\cdot): \mathbb{Y} \rightarrow \mathbb{T}$, is feasible if and only if it is (i) measurable and (ii) $s \leq t(y) \leq y$ for all $y \in \mathbb{Y}$.

\subsection{Agents' Utility Functions}

For each agent type $w \in W$, define

$$
K(w):=\{(y, \tau) \in K: y \in Y(w)\},
$$

and let $u(w, \cdot, \cdot, \cdot): K(w) \times \mathbb{R}_{+}^{k} \rightarrow \mathbb{R}$ denote the agent's utility function defined over 3 -tuples of income, tax liability, and public goods, $(y, \tau, z) \in K(w) \times \mathbb{R}_{+}^{k}$. We will assume agents' utility functions satisfy the following assumptions:

[A-3]

(1) (continuity): For each $w \in W, u(w, \cdot, \cdot, \cdot)$ is continuous

on $K(w) \times \mathbb{R}_{+}^{k}$.

(2) (measurability): For each $(y(\cdot), t(\cdot), z) \in S(Y(\cdot)) \times S(T(\cdot)) \times \mathbb{R}_{+}^{k}$, $u\left(\cdot, y(\cdot), t(y(\cdot), z)\right.$ is $\Sigma$-measurable. ${ }^{7}$

(3) (integrability): There exists a $\eta$-integrable function $\psi(\cdot): W \rightarrow \mathbb{R}$ such that for each $(y(\cdot), t(\cdot), z) \in S(Y(\cdot)) \times S(T(\cdot)) \times \mathbb{R}_{+}^{k}$, $|u(w, y(w), t(y(w)), z)| \leq \psi(w)$ a.e. $[\eta] .{ }^{8}$

(4) (monotonicity): For each $(w, y, z) \in W \times Y(w) \times \mathbb{R}_{+}^{k}$, $u(w, y, \cdot z)$ is strictly decreasing on $T(y)$.

\footnotetext{
${ }^{7}$ The function $u(\cdot, y(\cdot), t(y(\cdot), z)$ is $\Sigma$-measurable iff for any Borel measurable subset $E$ of the real numbers $\mathbb{R}$,$$
\{w \in W: u(w, y(w), t(y(w), z) \in E\} \in \Sigma
$$

${ }^{8} \psi(\cdot)$ is $\eta$-integrable if $\psi(\cdot): W \rightarrow \mathbb{R}$

$$
\int_{W}|\psi(w)| d \eta(w)<\infty
$$
}

We thank Guy Laroque for pointing out the need for assumption [A-3](3). 
We shall also assume that agents' preferences are such that leisure is essential; that is, we shall assume that in order for an agent to achieve a utility level at or above his reservation level, income must be accompanied by some positive amount of leisure. Stated formally, we have:

[A-4] (essentiality of leisure): For all agent types $w \in W$, $u(w, 0,0, z)>u(w, m(w), \tau, z)$ for all $(\tau, z) \in T(m(w)) \times \mathbb{R}_{+}^{k}$.

Under [A-4], if a type $w$ agent chooses the maximum level of income possible for his type, $m(w)$, then no matter what the tax liability $\tau$ or level of public goods $z$, the agent's utility, $u(w, m(w), \tau, z)$, is less than his reservation level, $u(w, 0,0, z)$. Thus, in order for a type $w$ agent to achieve a utility level at or above his reservation level, the agent must choose an income level strictly less than the maximum level attainable for his type - and therefore, the agent must choose a positive level of leisure. Assumption [A-4] thus guarantees that a type $w$ agent's optimal income choice will be in the income interval given by

$$
\{y \in \mathbb{Y}: 0 \leq y<m(w)\}
$$

a subset of the feasible income interval $Y(w):=\{y \in \mathbb{Y}: 0 \leq y \leq m(w)\}$.

Figure 1 below depicts the indifference curves corresponding to a type $w$ agent, given public goods vector $z$, for a utility function, $u(\cdot, \cdot, \cdot, \cdot)$, satisfying assumption [A-4]. Note that in Figure 1 the agent's utility function, $u(w, \cdot, \cdot, z)$, is defined over income and tax liability pairs in

$$
K(w):=\{(y, \tau) \in K: y \in K(w)\} .
$$

More importantly, note that in Figure 1 indifference curve 1, passing through the income and tax liability pair $(m(w), s)$, lies everywhere below (in terms of preference direction) indifference curve 2 , passing through the income and tax liability pair $(0,0)$. Thus, the preferences illustrated in Figure 1 satisfy assumption [A-4]. Note that in Figure 1, the vertical axis, the $\tau$ axis, is the tax liability axis, while the horizontal 
axis, the $y$ axis, is the income axis.

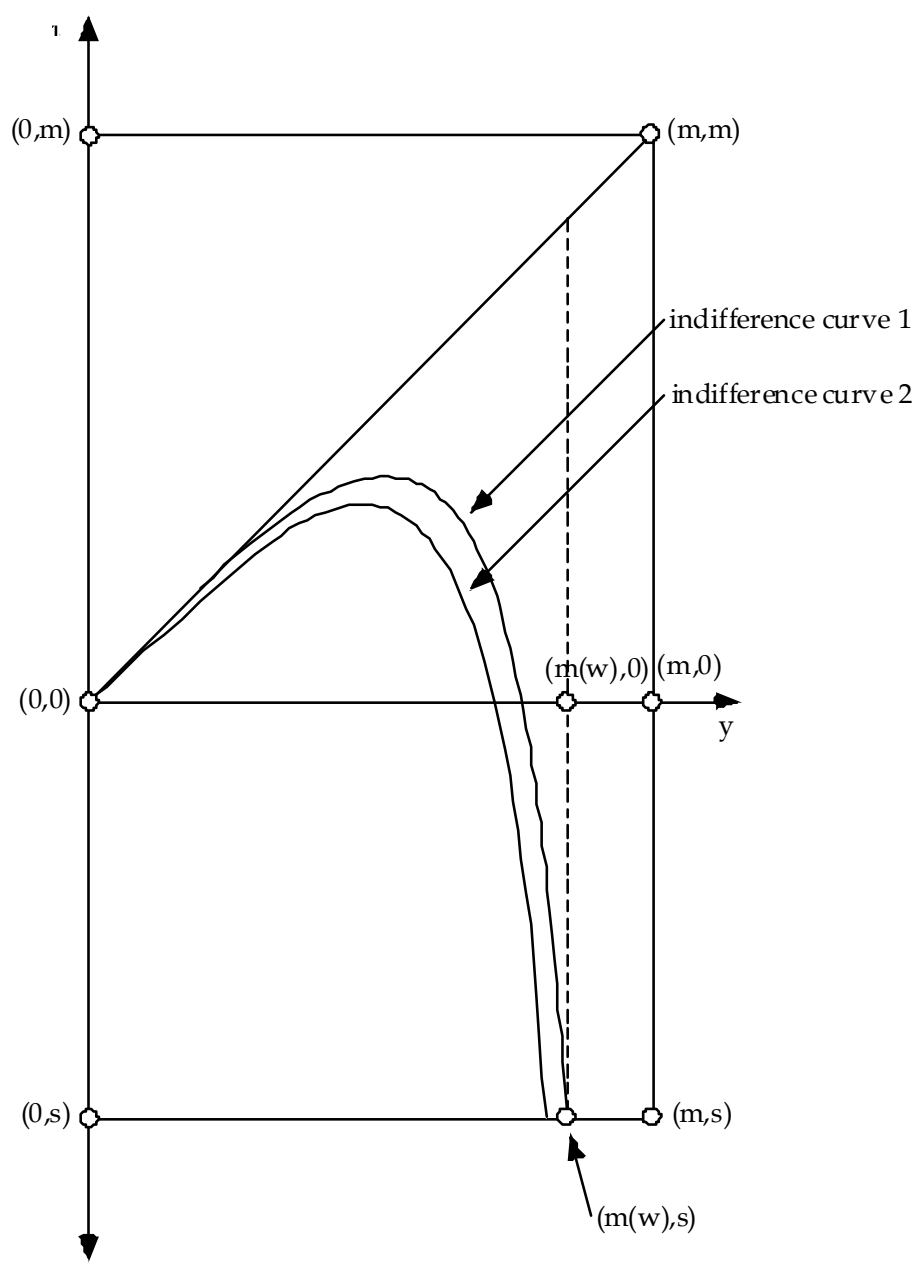

Figure 1

Example 2 (A Utility Function Satisfying the Essentiality of Leisure): As in Example 1, suppose the set of tax liabilities is given by an interval $\mathbb{T}=[0, m]$ and the set of agent types is given by an interval of positive ability parameters $W=$ $[a, b] \subset \mathbb{R}_{++}$. For each income and tax liability pair $(y, \tau) \in K$, let labor be given by $l=\frac{y}{w}$, and consumption by $c=y-\tau$. Letting $L>0$ denote the maximum amount of labor that can be supplied by any agent type. As before, we have $l \in[0, L]$ and $y \in[0, w L]$ (i.e., $m(w):=w L$ and $Y(w):=[0, w L]$ ). Suppose now that agents have preferences represented by a continuous utility function, $v(e, c, z)$, defined over leisure $e=L-l=L-\frac{y}{w} \in[0, L]$, consumption $c=y-\tau \in[0, w L]$, and public goods $z \in \mathbb{R}_{+}^{k}$, given by

$$
\begin{aligned}
& u(w, y, \tau, z)=v\left(L-\frac{y}{w}, y-\tau, z\right) \\
& =\left(L-\frac{y}{w}+\varepsilon\right)(\gamma(y-\tau)+\varepsilon)(z+\varepsilon) \text { for }(y, \tau) \in K(w) .
\end{aligned}
$$


Note that utility function $u(\cdot, \cdot, \cdot, \cdot)$ satisfies $[\mathrm{A}-3]$. Moreover, $u(\cdot, \cdot, \cdot, \cdot)$ will satisfy essentiality of leisure $([\mathrm{A}-4])$, if for all $(\tau, z) \in T(m(w)) \times[0, M]$,

$$
v(0,0, z)>v\left(\frac{m(w)}{w}, m(w)-\tau, z\right)=v(L, w L-\tau, z)
$$

It is easy to check that if the parameter $\gamma$ is such that $\gamma<1$, then $\left(^{*}\right)$ will be satisfied.

\subsection{Tax Design Problems with Public Goods}

We suppose that the government does not know each agent's type but can observe each agent's income and thus compute the resulting tax liability under any income tax function.

To begin, let $\mu(\cdot)$ be a finite measure defined on the measurable space of agent types $(W, \Sigma)$, equivalent to the measure $\eta(\cdot) .{ }^{9}$ The measure $\mu(\cdot)$ represents one possible welfare weighting scheme for agent types. ${ }^{10}$

The tax design problem with public goods can be stated as follows:

$$
\max _{(y(\cdot), t(\cdot), z)} \int_{W} u(w, y(w), t(y(w)), z) d \mu(w)
$$

subject to the constraints:

$$
\begin{gathered}
(y(\cdot), t(\cdot), z) \in S(Y(\cdot)) \times S(T(\cdot)) \times \mathbb{R}_{+}^{k}, \\
\text { for each } w \in W \text { and } y \in Y(w) \\
u(w, y(w), t(y(w)), z) \geq u(w, y, t(y), z), \\
\int_{W} t(y(w)) d \eta(w) \geq h(z)
\end{gathered}
$$

Expression (6) employs a utilitarian welfare function as is standard in the optimal income tax literature. Expression (7) is the feasibility constraint, while the inequalities given by (8) are the incentive compatibility constraints. Note that depending on the number of agent types, there can be uncountably many incentive compatibility constraints. Let $\Psi$ denote the set of feasible, incentive compatible income tax-public goods mechanisms. That is, $\Psi$ is the subset of mechanisms, $(y(\cdot), t(\cdot), z)$, contained in $S(Y(\cdot)) \times S(T(\cdot)) \times \mathbb{R}_{+}^{k}$ satisfying inequalities (8).

\footnotetext{
${ }^{9} \mu$ and $\eta$ are equivalent if they have the same sets of measure zero. Thus, $\mu$ and $\eta$ are equivalent if $\mu$ is absolutely continuous with respect to $\eta$ and $\eta$ is absolutely continuous with respect $\mu$.

${ }^{10}$ For a utilitarian welfare function, changing the measure $\mu$ can also be viewed as changing the cardinal representation of the underlying ordinal utilities. We thank John Ledyard and Leo Hurwicz for reminding us of this.
} 
The inequality constraint given in expression (9) is the financing constraint. It requires that any feasible income tax-public goods mechanism $(y(\cdot), t(\cdot), z)$ generate total tax revenues sufficient to cover the cost of providing public goods $z$. Denote by $\Pi$ the subset of income tax-public goods mechanisms, $(y(\cdot), t(\cdot), z)$, contained in $S(Y(\cdot)) \times S(T(\cdot)) \times \mathbb{R}_{+}^{k}$ satisfying inequalities $(9)$.

The tax design problem with public goods can be stated compactly as

$$
\max _{(y(\cdot), t(\cdot), z) \in \Psi \cap \Pi} \int_{W} u(w, y(w), t(y(w)), z) d \mu(w) .
$$

\subsection{Definitions: Implementation, Optimality, and Budget Balancedness}

Definition 1 (Implementation): We say that an income tax-public goods mechanism

$$
(y(\cdot), t(\cdot), z) \in S(Y(\cdot)) \times S(T(\cdot)) \times \mathbb{R}_{+}^{k}
$$

implements income tax function $t(\cdot)$ and finances public goods $z$ if and only if

$$
(y(\cdot), t(\cdot), z) \in \Psi \cap \Pi \text {. }
$$

Definition 2 (Second Best Pareto Optimality): We say that a income tax-public goods mechanism

$$
(y(\cdot), t(\cdot), z) \in S(Y(\cdot)) \times S(T(\cdot)) \times \mathbb{R}_{+}^{k}
$$

is Pareto optimal if and only if there does not exist another income tax-public goods mechanism $\left(y^{\prime}(\cdot), t^{\prime}(\cdot), z^{\prime}\right) \in S(Y(\cdot)) \times S(T(\cdot)) \times \mathbb{R}_{+}^{k}$ such that

$$
u\left(w, y^{\prime}(w), t^{\prime}(w), z^{\prime}\right) \geq u(w, y(w), t(w), z) \text { a.e. }[\eta]
$$

and

$$
u\left(w, y^{\prime}(w), t^{\prime}(w), z^{\prime}\right)>u(w, y(w), t(w), z) \text { for all } w \in E, E \in \Sigma \text { and } \eta(E)>0
$$

Definition 3 (Budget Balancedness): We say that a income tax-public goods mechanism

$$
(y(\cdot), t(\cdot), z) \in S(Y(\cdot)) \times S(T(\cdot)) \times \mathbb{R}_{+}^{k}
$$

satisfies budget balancedness if and only if

$$
\int_{W} t(y(w)) d \eta(w)=h(z)
$$

The following Proposition gives sufficient conditions for Pareto optimality. The proof is straightforward. 
Proposition 1 (Sufficient Conditions for Efficiency): If the mechanism

$$
(y(\cdot), t(\cdot), z) \in S(Y(\cdot)) \times S(T(\cdot)) \times \mathbb{R}_{+}^{k}
$$

solves the design problem ((6)-(9)) for some finite measure $\mu$ equivalent to the measure $\eta$, then it is Pareto optimal.

By Proposition 1, any income tax-public goods mechanism $\left(y^{*}(\cdot), t^{*}(\cdot), z^{*}\right) \in$ $\Psi \cap \Pi$ solving the design problem $((6)-(9))$ is Pareto optimal. Moreover, since $\left(y^{*}(\cdot), t^{*}(\cdot), z^{*}\right) \in \Pi$, the income tax function $t^{*}(\cdot)$ finances the vector of public goods $z^{*}$ under optimal income function $y^{*}(\cdot)$. Berliant and Page (2001) show that problem ((6)-(9)) has a solution. Here we address the more difficult question: Does there exist a Pareto optimal income tax-public goods mechanism satisfying budget balancedness?

\section{Main Results on Optimality and Budget Bal- ancedness}

By Theorem 1 in Berliant and Page (2001), under assumptions [A-1] to [A-4] there exists a Pareto optimal income tax and public goods mechanism, provided the set of incentive compatible and budget feasible mechanisms, $\Psi \cap \Pi$, is nonempty. In Theorems 1 and 2 below, we identify additional conditions - capacity constrained single crossing and the atomless condition - each of which guarantees the existence of a budget balancing, Pareto optimal income tax and public goods mechanism.

\subsection{Capacity Constrained Single Crossing and Budget Bal- ancedness}

Our first result states that if agents' utility functions satisfy the capacity constrained single crossing condition, then there exists a Pareto optimal income tax-public goods mechanism satisfying budget balancedness.

To begin, assume that,

[A-5] $\left\{\begin{array}{l}\text { the set of agent types } W \text { is given by } \\ \text { a subset of the real numbers equipped with the usual ordering. }\end{array}\right.$

We say that agents' utility functions satisfy the capacity constrained single crossing condition

[A-6] $\quad$ (1) $K(w) \subseteq K\left(w^{\prime}\right)$, and

if for all $z \in \mathbb{R}_{+}^{k}, w^{\prime}>w$ implies that

(2) for all $(y, \tau)$ and $\left(y^{\prime}, \tau^{\prime}\right)$ in $K(w)$ with $y^{\prime}>y$,

(a) $u\left(w, y^{\prime}, \tau^{\prime}, z\right) \geq u(w, y, \tau, z) \Rightarrow u\left(w^{\prime}, y^{\prime}, \tau^{\prime}, z\right) \geq u\left(w^{\prime}, y, \tau, z\right)$, and

(b) $u\left(w, y^{\prime}, \tau^{\prime}, z\right)>u(w, y, \tau, z) \Rightarrow u\left(w^{\prime}, y^{\prime}, \tau^{\prime}, z\right)>u\left(w^{\prime}, y, \tau, z\right)$. 
Example 3 (A Utility Function Satisfying the Capacity Constrained Single Crossing): As in Example 2, suppose that the set of tax liabilities is given by an interval $\mathbb{T}=[0, m]$ and that the set of agent types is given by an interval of positive ability parameters $W=[a, b] \subset \mathbb{R}_{++}$. Recall that for each income and tax liability pair $(y, \tau) \in K$, labor is given by $l=\frac{y}{w}$, while consumption is given by $c=y-\tau$. Now suppose that agents have preferences represented by a twice continuously differentiable utility function, $v(e, c, z)$, defined over leisure $e=L-l=L-\frac{y}{w} \in[0, L]$, consumption $c=y-\tau \in[0, w L]$, and public goods $z \in \mathbb{R}_{+}^{k}$, given by

$$
u(w, y, \tau, z)=v\left(L-\frac{y}{w}, y-\tau, z\right) .
$$

As in Example 2, $v(\cdot, \cdot, \cdot)$ might take the specific form,

$$
v\left(L-\frac{y}{w}, y-\tau, z\right)=\left(L-\frac{y}{w}+\varepsilon\right)(\gamma(y-\tau)+\varepsilon)(z+\varepsilon) \text { for }(y, \tau) \in K(w) .
$$

Also, recall from Example 2 that labor $l=\frac{y}{w} \in[0, L]$ and income $y \in[0, w L]$ where $L>0$ is the maximum amount of labor that can be supplied by any agent type (i.e., $m(w):=w L$ and $Y(w):=[0, w L])$.

If $v(\cdot, \cdot, \cdot)$ satisfies the following derivative conditions

$$
\text { (i) } \frac{\partial v}{\partial e}>0, \frac{\partial v}{\partial c}>0 \text {, (ii) } \frac{\partial^{2} v}{\partial e^{2}} \leq 0 \text {, and (iii) } \frac{\partial^{2} v}{\partial e \partial c} \geq 0 \text {, }
$$

then

$$
u(w, y, \tau, z)=v\left(L-\frac{y}{w}, y-\tau, z\right)
$$

satisfies capacity constrained single crossing [A-6]. To see this consider the following:

Let $(y, \tau)$ and $\left(y^{\prime}, \tau^{\prime}\right)$ in $K(w)$ be such that $y^{\prime}>y$, and suppose that

$$
\begin{array}{r}
v\left(L-\frac{y^{\prime}}{w}, y^{\prime}-\tau^{\prime}, z\right) \geq v\left(L-\frac{y}{w}, y-\tau, z\right),(*) \\
\text { but for some } w^{\prime}>w \\
v\left(L-\frac{y^{\prime}}{w^{\prime}}, y^{\prime}-\tau^{\prime}, z\right)<v\left(L-\frac{y}{w^{\prime}}, y-\tau, z\right) .(* *)
\end{array}
$$

It follows from $(*)$ and $(* *)$ and the continuity of the utility function that for some $\bar{w} \in\left[w, w^{\prime}\right]$

$$
v\left(L-\frac{y^{\prime}}{\bar{w}}, y^{\prime}-\tau^{\prime}, z\right)=v\left(L-\frac{y}{\bar{w}}, y-\tau, z\right) .(* * *)
$$

Since $L-\frac{y^{\prime}}{w}<L-\frac{y}{w},(* * *)$ and $\frac{\partial v}{\partial c}>0$ imply that $y^{\prime}-\tau^{\prime}>y-\tau$. Given $\frac{\partial v}{\partial e}>0$ and $\frac{\partial^{2} v}{\partial e^{2}} \leq 0$, we know that

$$
\frac{\partial v}{\partial e}\left(L-\frac{y^{\prime}}{w}, y-\tau, z\right) \geq \frac{\partial v}{\partial e}\left(L-\frac{y}{w}, y-\tau, z\right) \text { for all } w
$$


Also, since $\frac{\partial^{2} v}{\partial e \partial c} \geq 0, y^{\prime}-\tau^{\prime}>y-\tau$ implies that

$$
\frac{\partial v}{\partial e}\left(L-\frac{y^{\prime}}{w}, y^{\prime}-\tau^{\prime}, z\right) \geq \frac{\partial v}{\partial e}\left(L-\frac{y^{\prime}}{w}, y-\tau, z\right) \text { for all } w .
$$

Therefore,

$$
\frac{\partial v}{\partial e}\left(L-\frac{y^{\prime}}{w}, y^{\prime}-\tau^{\prime}, z\right) \geq \frac{\partial v}{\partial e}\left(L-\frac{y}{w}, y-\tau, z\right) \text { for all } w,
$$

contradicting $(* *)$.

Suppose now that

$$
\begin{gathered}
v\left(L-\frac{y^{\prime}}{w}, y^{\prime}-\tau^{\prime}, z\right)>v\left(L-\frac{y}{w}, y-\tau, z\right), \\
\text { but for some } w^{\prime}>w \\
v\left(L-\frac{y^{\prime}}{w^{\prime}}, y^{\prime}-\tau^{\prime}, z\right) \leq v\left(L-\frac{y}{w^{\prime}}, y-\tau, z\right) .
\end{gathered}
$$

Again, we have for some $\bar{w} \in\left[w, w^{\prime}\right]$

$$
v\left(L-\frac{y^{\prime}}{\bar{w}}, y^{\prime}-\tau^{\prime}, z\right)=v\left(L-\frac{y}{\bar{w}}, y-\tau, z\right) .
$$

Repeating the argument above, we obtain a contradiction.

Thus, if agents' utility functions are given by

$$
u(w, y, \tau, z)=v\left(L-\frac{y}{w}, y-\tau, z\right), w \in[a, b],
$$

and if the derivative conditions, (i), (ii), and (iii), above are satisfied, then capacity constrained single crossing, [A-6], holds. Moreover, if $v(\cdot, \cdot, \cdot)$ is of the form,

$$
v\left(L-\frac{y}{w}, y-\tau, z\right)=\left(L-\frac{y}{w}+\varepsilon\right)(\gamma(y-\tau)+\varepsilon)(z+\varepsilon)
$$

with $0<\gamma<1$, then the essentiality of leisure, [A-4], also holds (see Example 2).

Our main result on capacity constrained single crossing and budget balancedness is the following:

Theorem 1 (The Capacity Constrained Single Crossing Condition and Budget Balancedness):

Suppose that $[A-1]-[A-4]$ hold and suppose that $\Psi \cap \Pi \neq \emptyset$.

If the set of agent types $W$ is given by a subset of the real numbers equipped with the usual ordering, that is if [A-5] holds, and if the capacity constrained single crossing condition [A-6] holds, then there exists an optimal income tax-public goods mechanism that is budget balancing. In particular, for any finite measure $\mu$ defined on the space of agent types $(W, \Sigma)$ equivalent to $\eta$ there exists a mechanism $\left(y^{*}(\cdot), t^{*}(\cdot), z^{*}\right) \in \Psi \cap \Pi$

$$
\text { maximizing } \int_{W} u(w, y(w), t(y(w)), z) d \mu(w) \text { over } \Psi \cap \Pi
$$

such that

$$
\int_{W} t^{*}\left(y^{*}(w)\right) d \eta(w)=h\left(z^{*}\right)
$$




\subsection{The Atomless Condition and Budget Balancedness}

A subset of agent types $E \in \Sigma$ is an atom in the measure space $(W, \Sigma, \eta)$ if $\eta(E)>0$ and for all $F \in \Sigma$ such that $F \subseteq E$, either $\eta(F)=0$ or $\eta(E-F)=0$. We say that $(W, \Sigma, \eta)$ satisfies the atomless condition if

$$
[\mathrm{A}-7](W, \Sigma, \eta) \text { contains no atoms. }
$$

Example 4 (Densities and the Atomless Condition): Suppose the measure space of agent types, $(W, \Sigma, \eta)$, is specified as follows: $W=\mathbb{R}^{m}, \Sigma$ is the Lebesgue $\sigma$-field, and $\eta$ is specified via a density function $f$; that is, for $E \in \Sigma$,

$$
\eta(E)=\int_{E} f(w) d \lambda(w)
$$

where $\lambda$ is Lebesgue measure on $\mathbb{R}^{m}$. Then $(W, \Sigma, \eta)$ contains no atoms - and therefore, $(W, \Sigma, \eta)$ satisfies the atomless condition.

Our main result on the atomless condition and budget balancedness is the following:

Theorem 2 (The Atomless Condition and Budget Balancedness):

Suppose that $[A-1]-[A-4]$ hold and suppose that $\Psi \cap \Pi \neq \emptyset$.

If the measure space of agent types $(W, \Sigma, \eta)$ contains no atoms, so that [A-7] holds, then there exists an optimal income tax-public goods mechanism that is budget balancing. In particular, for any finite measure $\mu$ defined on the space of agent types $(W, \Sigma)$ equivalent to $\eta$ there exists a mechanism $\left(y^{*}(\cdot), t^{*}(\cdot), z^{*}\right) \in \Psi \cap \Pi$

$$
\text { maximizing } \int_{W} u(w, y(w), t(y(w)), z) d \mu(w) \text { over } \Psi \cap \Pi
$$

such that

$$
\int_{W} t^{*}\left(y^{*}(w)\right) d \eta(w)=h\left(z^{*}\right)
$$

\subsection{The Intuition}

\subsubsection{Pictures of the Problem and the Solution}

In order to better understand the problem of budget balancedness and the intuition behind our results, we begin by considering the tax and income possibility set (TIPS). This set contains all possible aggregate income and tax revenue pairs attainable under some incentive compatible income tax and public goods mechanism, given a particular income tax function and public goods pair. For example, Figure 2 below depicts the tax and income possibilities set, the TIPS, corresponding to income tax function and public goods pair $(t(\cdot), z)$, where each point in the set is generated by some income tax and public goods mechanism $(y(\cdot), t(\cdot), z) \in \Psi$. Thus, given $(t(\cdot), z)$ the aggregate 
income and tax pairs, $(Y, T)$, contained in TIPS are generated by varying the direct income function, $y(\cdot)$, so that $(y(\cdot), t(\cdot), z)$ is incentive compatible (i.e., contained in $\Psi)$.

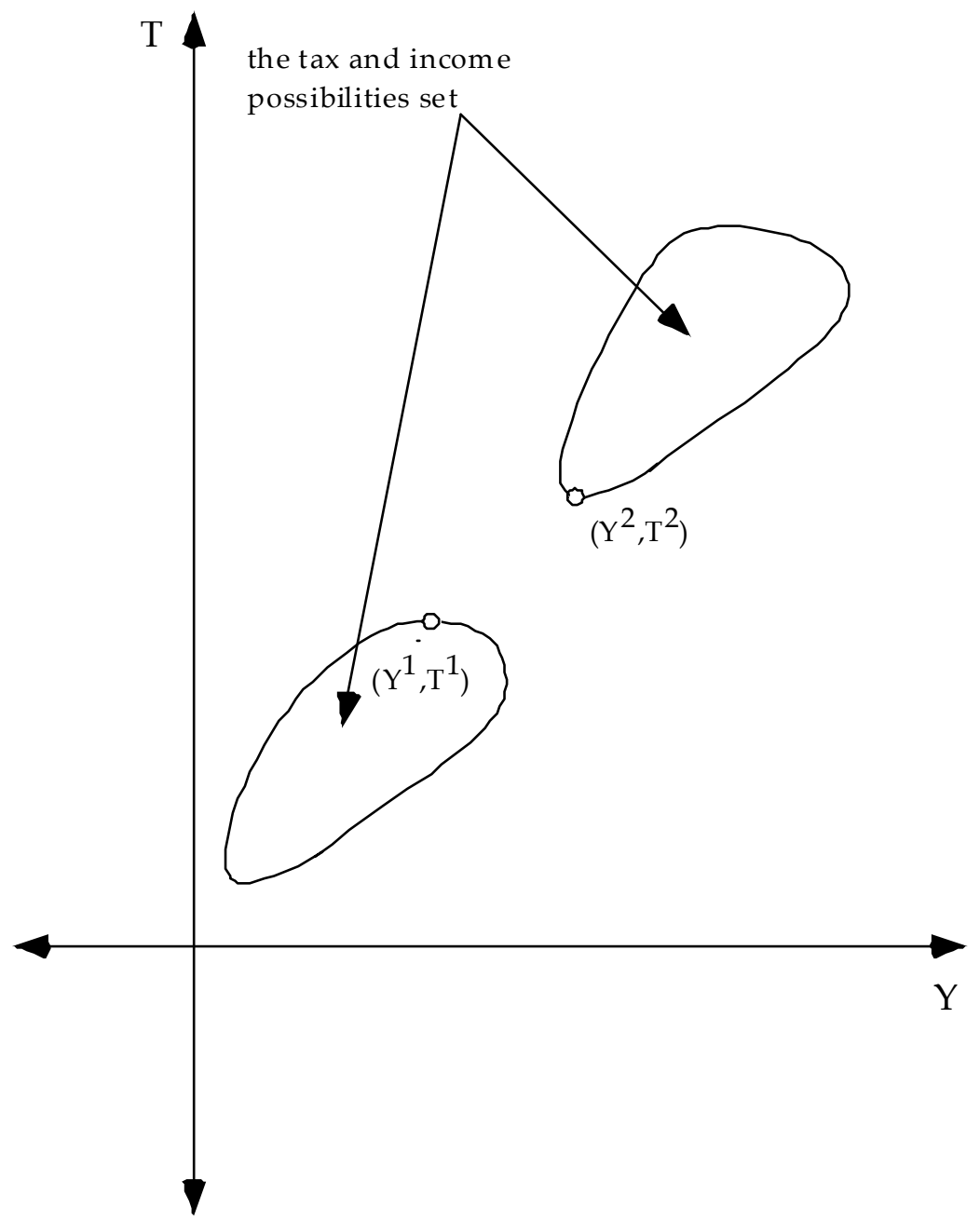

Figure 2

In Figure 2, the vertical axis, the $T$ axis, is the aggregate tax revenue axis, while the horizontal axis, the $Y$ axis, is the aggregate income axis.

In Figure 2, corresponding to the point $\left(Y^{1}, T^{1}\right)$ there is a mechanism $\left(y^{1}(\cdot), t(\cdot), z\right) \in$ $\Psi$ such that

$$
Y^{1}=\int_{W} y^{1}(w) d \eta(w) \text { and } T^{1}=\int_{W} t\left(y^{1}(w)\right) d \eta(w)
$$

and corresponding to $\left(Y^{2}, T^{2}\right)$ there is a mechanism $\left(y^{2}(\cdot), t(\cdot), z\right) \in \Psi$ such that

$$
Y^{2}=\int_{W} y^{2}(w) d \eta(w) \text { and } T^{2}=\int_{W} t\left(y^{2}(w)\right) d \eta(w)
$$

The problem of budget balancedness arises if the cost of providing public goods $z$, given by $h(z)$, is such that for all aggregate income and tax revenue pairs, $(Y, T)$, 
contained in TIPS either

$$
T<h(z) \text { or } h(z)<T .
$$

Figure 3 depicts the problem.

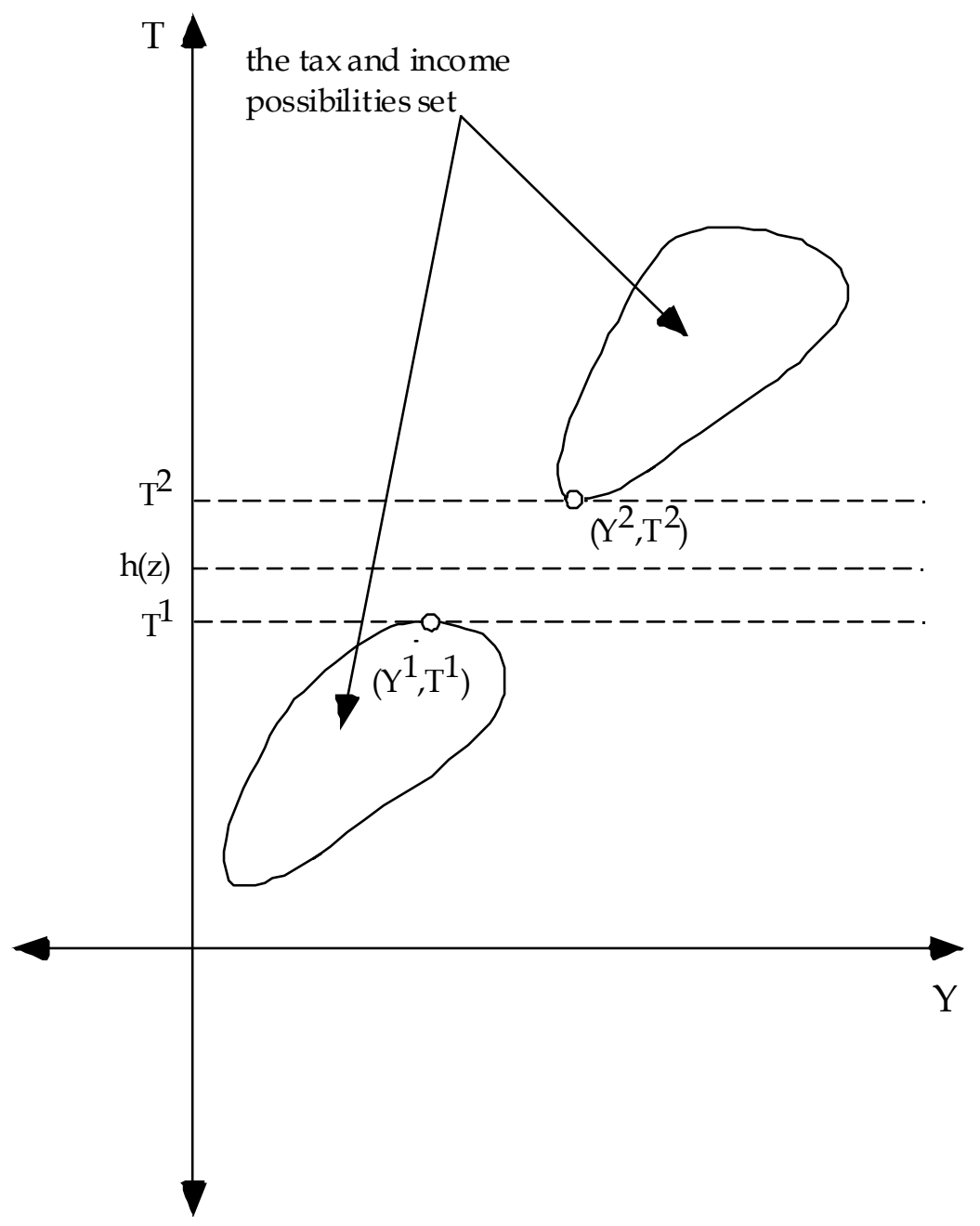

Figure 3

In Figure 3, there does not exist an aggregate income and tax pair, $(Y, T)$, contained in TIPS such that $T=h(z)$. Note that the mechanism $\left(y^{2}(\cdot), t(\cdot), z\right)$ generating $\left(Y^{2}, T^{2}\right)$ satisfies the financing constraint. Thus, $\left(y^{2}(\cdot), t(\cdot), z\right) \in \Psi \cap \Pi$. However, the mechanism $\left(y^{1}(\cdot), t(\cdot), z\right)$ generating $\left(Y^{1}, T^{1}\right)$ does not satisfy the financing constraint. Thus, $\left(y^{1}(\cdot), t(\cdot), z\right) \notin \Pi$, and therefore, $\left(y^{1}(\cdot), t(\cdot), z\right) \notin \Psi \cap \Pi$. Finally, note that if $(y(\cdot), t(\cdot), z) \notin \Pi$ for all direct income functions, $y(\cdot)$, such that $(y(\cdot), t(\cdot), z)$ is incentive compatible (i.e., contained in $\Psi$ ), then the income tax function and public goods pairs $(t(\cdot), z)$ would not be a viable solution candidate for the optimal income tax problem.

With these pictures in mind, it is easy to see that if the projection of the tax and income possibilities set, TIPS, onto the aggregate income tax axis is convex, then 
the problem of budget balancedness goes away. We call this projection condition tax convexity. Thus, if the TIPS corresponding to an income tax function and public goods pair $(t(\cdot), z)$ is tax convex, then budget balancedness can be achieved under some direct income function $y(\cdot)$ such that $(y(\cdot), t(\cdot), z)$ is incentive compatible. This is the intuition underlying Theorem 4 below. Figure 4 depicts a tax convex TIPS corresponding to income tax function and public goods pair $(t(\cdot), z)$.

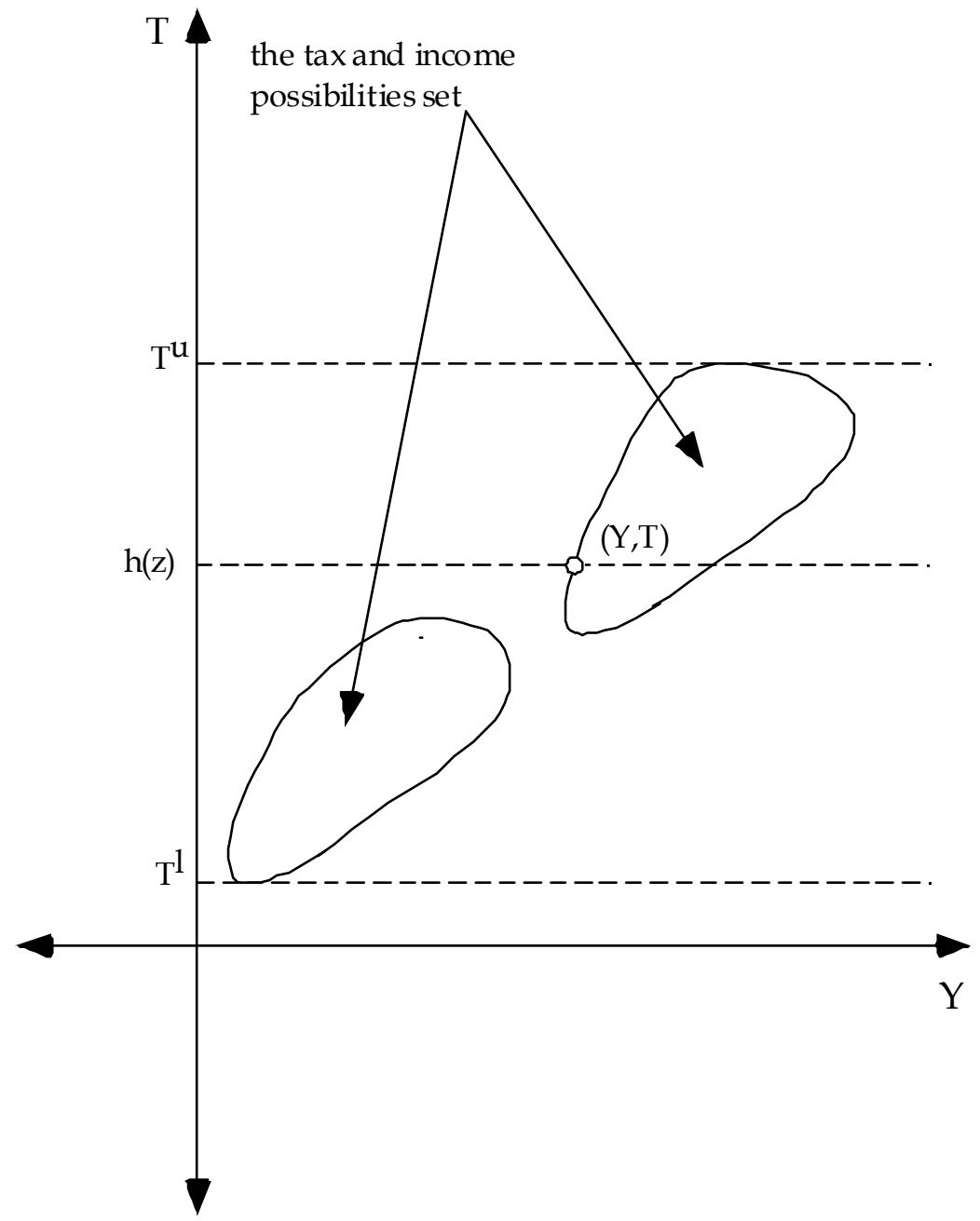

Figure 4

Note that in this case the projection of the TIPS onto the aggregate income tax axis (the $T$-axis) is given by the interval $\left[T^{l}, T^{u}\right]$, and is therefore convex. Also note that the projection of the TIPS onto the aggregate income axis (the $Y$-axis) is not convex. Moreover, note that for the aggregate income and tax pair $(Y, T)$ contained in the TIPS and depicted in Figure $4, T=h(z)$. Thus for this given income tax function and public goods pair, $(t(\cdot), z)$, there is some direct income function $y(\cdot)$ such that 
$(y(\cdot), t(\cdot), z)$ is incentive compatible and such that

$$
Y=\int_{W} y(w) d \eta(w) \text { and } T=\int_{W} t(y(w)) d \eta(w)=h(z) .
$$

\subsubsection{The Intuition Behind the Solution}

We identify two conditions on the primitives of the model guaranteeing tax convexity of the TIPS: (i) the capacity constrained single crossing condition [A-6] (see Theorem 5), and (ii) the atomless condition [A-7] (see Theorem 6). The key ingredient which allows us to see the connection between single crossing and tax convexity and between atomlessness and tax convexity is a transformation of the optimal income tax problem ((6)-(9)) from a mechanism design problem to a menu design problem (see Corollary 1; also see Hammond (1979), Holmstrom (1984) and Page (1992)). It is in the transformed problem - the menu design problem - that we are able to identify conditions sufficient for tax convexity. The validity of this transformation rests upon a fundamental result characterizing all income tax-public goods mechanisms in $\Psi \cap \Pi$ in terms of revenue feasible menu and public goods pairs (see Theorem 3).

The basic idea is straightforward: rather than have the government choose an optimal income tax-public goods mechanism $\left(y^{*}(\cdot), t^{*}(\cdot), z^{*}\right) \in \Psi \cap \Pi$, instead, in the transformed problem, the government chooses an optimal menu-public goods pair and delegates choice from the menu to the agents. Each menu consists of a set of income and tax liability pairs. Figure 5 below illustrates the idea of a menu, as well as the choice problem faced by a type $w$ agent given menu and public goods pair $(C, z) \in \Lambda \times \mathbb{R}_{+}^{k}$. Here, $\Lambda$ denotes the feasible set of menus. In Figure 5 , the vertical axis, the $\tau$ axis, is the tax liability axis, while the horizontal axis, the $y$ axis, is the 
income axis.

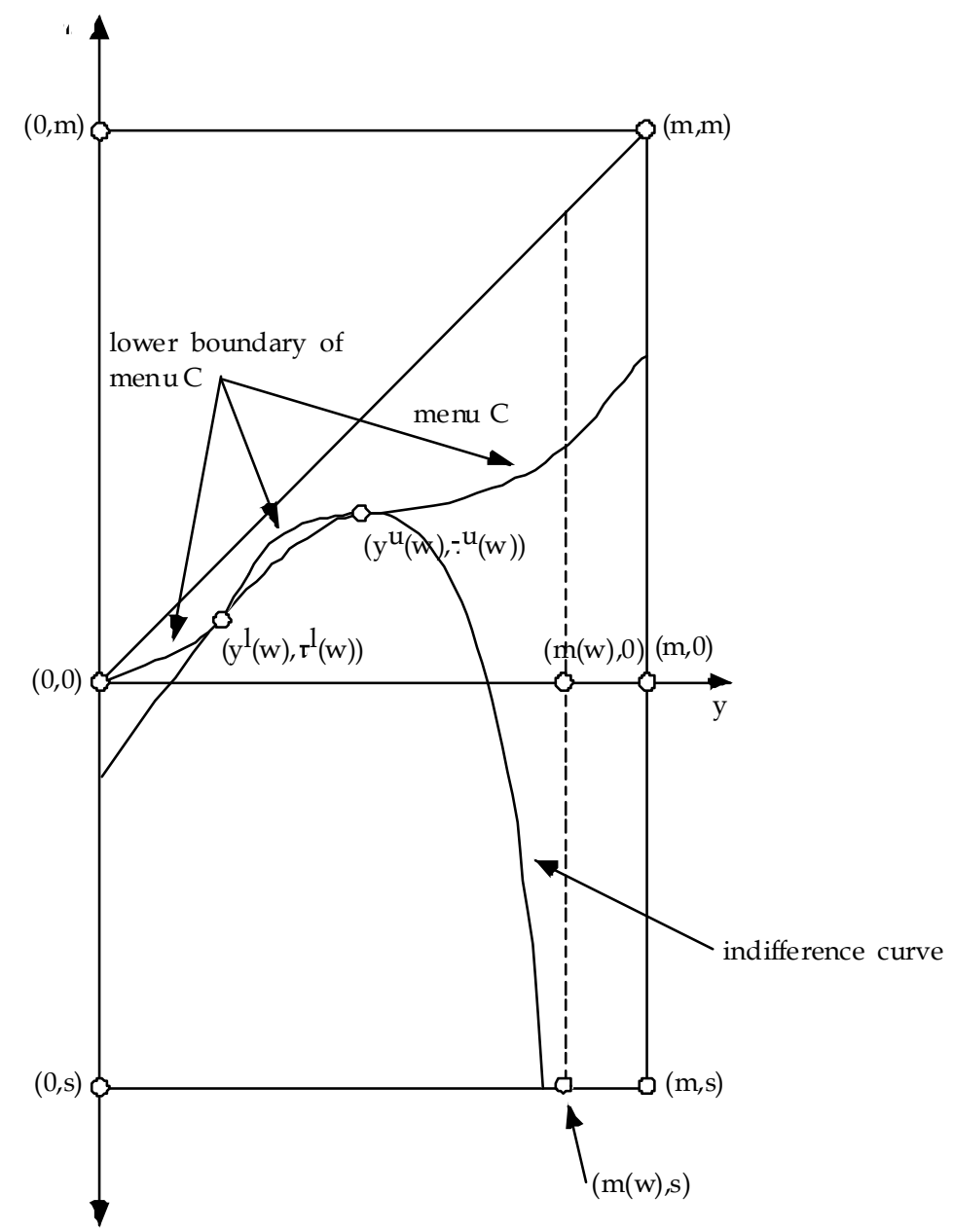

Figure 5

Given that utility is strictly decreasing in taxes, all agents, no matter what their types, will choose an optimal income and tax liability pair on the lower boundary of menu $C$. Letting $\Phi(w, C, z)$ denote the best responses (i.e., the optimal income and tax liability choices) of a type $w \in W$, given menu-public goods pair $(C, z) \in \Lambda \times \mathbb{R}_{+}^{k}$, we have in Figure 5,

$$
\Phi(w, C, z)=\left\{\left(y^{l}(w), \tau^{l}(w)\right),\left(y^{u}(w), \tau^{u}(w)\right)\right\}
$$

where each pair, $\left(y^{l}(w), \tau^{l}(w)\right)$ and $\left(y^{u}(w), \tau^{u}(w)\right)$, is on the lower boundary of menu $C$ and on the same type $w$ agent's indifference curve.

Note that no income and tax liability pairs along the lower boundary of menu $C$ between $\left(y^{l}(w), \tau^{l}(w)\right)$ and $\left(y^{u}(w), \tau^{u}(w)\right)$ are chosen by type $w$ agents. Thus, there is a gap in tax revenue from type $w$ agents between $\tau^{l}(w)$ and $\tau^{u}(w)$ - and depending on the distribution of agent types, such gaps can cause tax convexity to 
fail. We show in Theorem 5 below that if capacity constrained single crossing is satisfied, then such gaps can be eliminated, without altering the utility of any agent type and hence without breaking incentive compatibility. In particular, we show that tax revenue gaps can be eliminated by replacing menu $C$ with a menu $D$ where the lower boundary of menu $D$ between $\left(y^{l}(w), \tau^{l}(w)\right)$ and $\left(y^{u}(w), \tau^{u}(w)\right)$ is given by the indifference curve passing through $\left(y^{l}(w), \tau^{l}(w)\right)$ and $\left(y^{u}(w), \tau^{u}(w)\right)$. Figure 6 depicts the new menu $D$ equivalent to menu $C$ under capacity constrained single crossing.

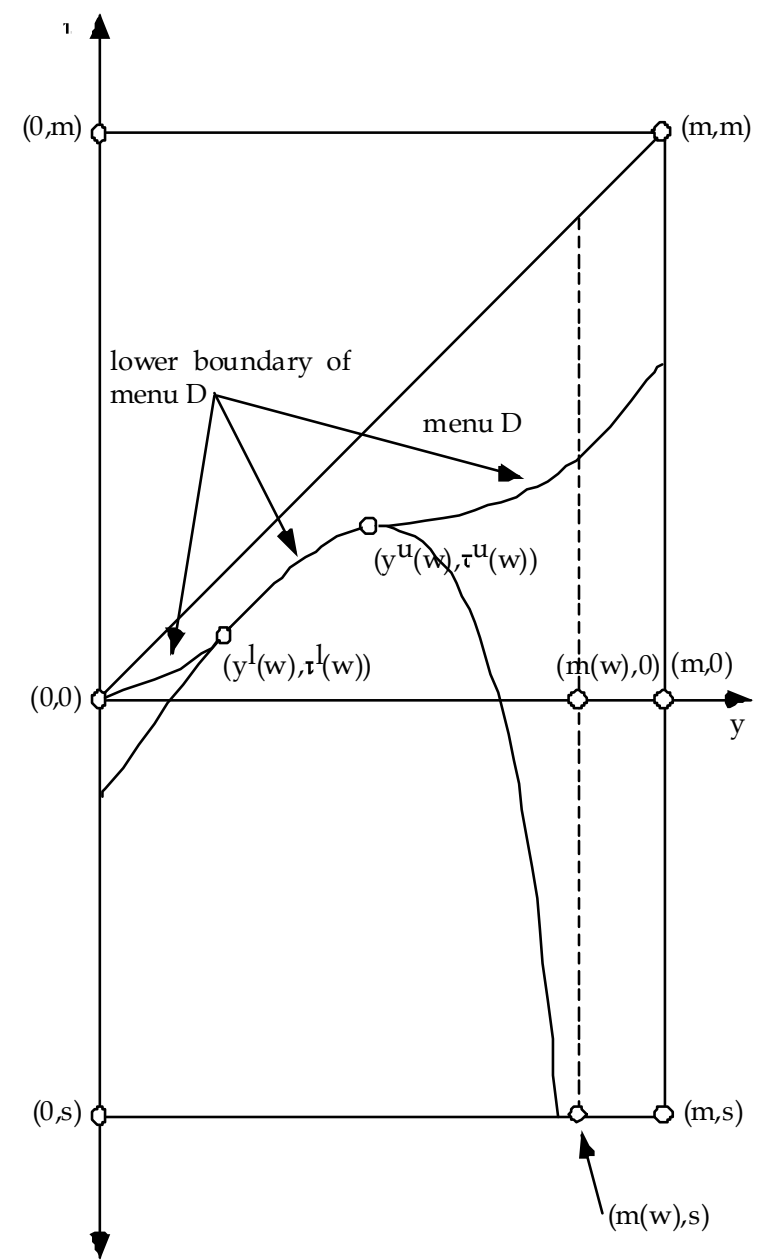

Figure 6

Under menu $D$, a type $w$ agent's best responses, $\Phi(w, D, z)$, include $\left(y^{l}(w), \tau^{l}(w)\right)$ and $\left(y^{u}(w), \tau^{u}(w)\right)$, as well as all the income and tax liability pairs, $(y, \tau)$ between $\left(y^{l}(w), \tau^{l}(w)\right)$ and $\left(y^{u}(w), \tau^{u}(w)\right)$ on the indifference curve containing $\left(y^{l}(w), \tau^{l}(w)\right)$ and $\left(y^{u}(w), \tau^{u}(w)\right)$. Note that a type $w$ agent's optimal utility under menu-public goods pair $(C, z) \in \Lambda \times \mathbb{R}_{+}^{k}$ is equal a type $w$ agent's optimal utility under menu-public goods pair $(D, z) \in \Lambda \times \mathbb{R}_{+}^{k}$. More importantly, note that under $(D, z) \in \Lambda \times \mathbb{R}_{+}^{k}$ there is no longer a tax revenue gap between $\tau^{l}(w)$ and $\tau^{u}(w)$. In the proof of Theorem 5 , we show that, in general, under capacity constrained single crossing, a menu which 
produces tax revenue gaps, for example menu $C$ in Figure 5, can always be replaced by a menu which does not produce tax revenue gaps, for example menu $D$ in Figure 6 - without altering agents' incentives or diminishing potential tax revenues. Moreover, we show in the proof of Theorem 5 that the TIPS corresponding to a menu-public goods pair which generates no tax revenue gaps is tax convex. Together, Theorem 4 and Theorem 5 imply Theorem 1.

In Theorem 6 we show that if the measure space of agent types is atomless (i.e., contains no atoms), then the TIPS corresponding to any menu-public goods pair is convex - and therefore tax convex. Here the basic intuition is that without atoms (i.e., without rare and significant agent types) the set of types is sufficiently rich and varied to guarantee that aggregate tax revenue gaps (as well as aggregate income gaps) get filled in via the optimizing behavior of agents. Together, Theorem 4 and Theorem 6 imply Theorem 2.

In the remaining sections of the paper, we carry out the formal analysis of the optimal income tax problem. First, we transform the optimal income tax problem from a mechanism problem to a menu problem (Theorem 3 and Corollary 1). Next, within the context of the transformed problem, we show that tax convexity implies budget balancedness (Theorem 4). Finally, we show that capacity constrained single crossing implies the tax convexity of the TIPS (Theorem 5), and we show that the atomless condition implies convexity of the TIPS (Theorem 6).

\section{Transforming the Optimal Income Tax Problem: From Mechanisms to Menus}

\subsection{Menus and Revenue Feasibility}

\subsubsection{Menus}

To begin, let $P_{f}(K)$ denote the collection of all nonempty closed subsets of

$$
K:=\{(y, \tau) \in \mathbb{Y} \times \mathbb{T}: y \geq \tau\} .
$$

Since $K$ is a compact metric space, $P_{f}(K)$ equipped with the Hausdorff metric $h$ is also a compact metric space (Berge (1963)).

Since the government cannot control or restrict an agent's income choice, any income and tax liability menu $C \in P_{f}(K)$ chosen by the government must be such that $\operatorname{proj}_{\mathbb{Y}}(C)=\mathbb{Y}$, where $\operatorname{proj}_{\mathbb{Y}}(C)$ denotes the projection of the closed set $C \subset \mathbb{Y} \times \mathbb{T}$ onto $\mathbb{Y}$. Hence menu choice must be restricted to the subset of menus $\Lambda$ given by

$$
\Lambda:=\left\{C \in P_{f}(K): \operatorname{proj}_{\mathbb{Y}}(C)=\mathbb{Y}\right\} .
$$

The set $\Lambda$ is nonempty (e.g., take the 45 degree line in $\mathbb{Y} \times \mathbb{T}$ ) and closed with respect to the Hausdorff metric. ${ }^{11}$ Thus, $(\Lambda, h)$ is a compact metric space.

\footnotetext{
${ }^{11}$ In particular, it is easy to show that if $\left\{C_{n}\right\}_{n} \subset \Lambda$ converges to $C \in P_{f}(K)$ under the metric $h$,
} 


\subsubsection{Delegated Choice and the Best Response Mapping}

Given a particular menu and public goods pair $(C, z) \in \Lambda \times \mathbb{R}_{+}^{k}$ chosen by the government, the resulting delegated choice problem for agents is given by

$$
\max _{(y, \tau) \in C \cap K(w)} u(w, y, \tau, z) .
$$

For each agent type $w \in W$, choice problem (15) has a solution. Let

$$
\widehat{u}(w, C, z):=\max _{(y, \tau) \in C \cap K(w)} u(w, y, \tau, z),
$$

and

$$
\Phi(w, C, z):=\{(y, \tau) \in C \cap K(w): u(w, y, \tau, z) \geq \widehat{u}(w, C, z)\} .
$$

Given menu and public goods pair $(C, z) \in \Lambda \times \mathbb{R}_{+}^{k}, \widehat{u}(w, C, z)$ is the maximal level of utility attainable by a type $w$ agent, while $\Phi(w, C, z)$ is the set of income and tax liability pairs from which the type $w$ agent must choose in order to attain utility level $\widehat{u}(w, C, z)$. Thus, the mapping $w \rightarrow \Phi(w, C, z)$ is the best response mapping.

Proposition 2 (Continuity Properties of the Maximal Utility Function and the Best Response Mapping):

(1) Under assumption [A-3], $\widehat{u}(w, \cdot, \cdot)$ is continuous on $\Lambda \times \mathbb{R}_{+}^{k}$ for each $w \in W$ (with respect to the product metric) and $\widehat{u}(\cdot, C, z)$ is $\Sigma$-measurable on $W$ for each $(C, z) \in \Lambda \times \mathbb{R}_{+}^{k}$.

(2) $\Phi(w, C, z)$ is nonempty and compact for each $(w, C, z) \in W \times \Lambda \times \mathbb{R}_{+}^{k}$. Moreover, $\Phi(w, \cdot, \cdot)$ is upper semicontinuous on $\Lambda \times \mathbb{R}_{+}^{k}$ for each $w \in W$ (with respect to the product metric) and $\Phi(\cdot, \cdot, \cdot)$ is $\Sigma \times B(\Lambda) \times B\left(\mathbb{R}_{+}^{k}\right)$-measurable on $W \times \Lambda \times \mathbb{R}_{+}^{k} \cdot{ }^{12}$

Under the assumption of essentiality of leisure (i.e.,[A-4]), The proof of Proposition 2 follows directly from Propositions 4.1 and 4.2 in Page (1992).

Let

$\Sigma(C, z):=\{(y(\cdot), \tau(\cdot)) \in M(W, \mathbb{Y}) \times M(W, \mathbb{T}):(y(w), \tau(w)) \in \Phi(w, C, z) \forall w \in W\}$

$\Sigma(C, z)$ is the set of all measurable selections from the best response mapping, $w \rightarrow \Phi(w, C, z)$, given menu, public goods pair $(C, z) \in \Lambda \times \mathbb{R}_{+}^{k}$. By the KuratowskiRyll-Nardzewski Selection Theorem (see Aliprantis and Border (1999), p. 567),

then $\operatorname{proj}_{\mathbb{Y}}(C)=\mathbb{Y}$.

${ }^{12} B(\Lambda)$ denotes the Borel $\sigma$-field in the compact metric space $(\Lambda, h)$ and $B\left(\mathbb{R}_{+}^{k}\right)$ the Borel $\sigma$-field in $\mathbb{R}_{+}^{k} . \Phi(\cdot, \cdot, \cdot)$ is $\Sigma \times B(\Lambda) \times B\left(\mathbb{R}_{+}^{k}\right)$-measurable iff for each closed subset $E$ of $\mathbb{Y} \times \mathbb{T}$, the subset of 3-tuples $(w, C, z)$ in $W \times \Lambda \times \mathbb{R}_{+}^{k}$ such that

$$
\Phi(w, C, z) \cap E \neq \emptyset
$$

is contained in $\Sigma \times B(\Lambda) \times B\left(\mathbb{R}_{+}^{k}\right)$ (see Himmelberg (1975)). 
for any $(C, z) \in \Lambda \times \mathbb{R}_{+}^{k}, \Sigma(C, z)$ is nonempty. Because each measurable selection, $(y(\cdot), \tau(\cdot)) \in \Sigma(C, z)$, is defined directly on the set of agent types $W$, each $(y(\cdot), \tau(\cdot)) \in \Sigma(C, z)$ is a direct mechanism. Thus, $\Sigma(C, z)$ is the set of all incentive compatible direct mechanisms given menu public goods pair $(C, z) \in \Lambda \times \mathbb{R}_{+}^{k}$.

\subsubsection{Revenue Feasibility}

Definition 4 (Revenue Feasibility): A menu and public goods pair $(C, z) \in \Lambda \times \mathbb{R}_{+}^{k}$ is revenue feasible if there exists a direct mechanism $(y(\cdot), \tau(\cdot)) \in \Sigma(C, z)$ such that

$$
\int_{W} \tau(w) d \eta(w) \geq h(z)
$$

Let $R$, a subset of $\Lambda \times \mathbb{R}_{+}^{k}$, denote the set of all revenue feasible menu, public goods pairs.

Proposition 3 (Compactness of the Set of Revenue Feasible Menu, Public Goods Pairs): $R$ is a compact subset of $\Lambda \times \mathbb{R}_{+}^{k}$.

\subsection{The Equivalence of Mechanisms and Menus}

Theorem 3 (The Equivalence of Mechanisms and Menus):

Suppose $[A-1]-[A-4]$ hold.

1. Given any income tax-public goods mechanism $(y(\cdot), t(\cdot), z) \in \Psi \cap \Pi$, there exists a pair $(C, z) \in R$ such that

$$
(y(w), t(y(w))) \in \Phi(w, C, z) \text { for all } w \in W .
$$

2. Given any pair $(C, z) \in R$, there exists a income tax-public goods mechanism $(y(\cdot), t(\cdot), z) \in \Psi \cap \Pi$ such that

$$
(y(w), t(y(w))) \in \Phi(w, C, z) \text { for all } w \in W .
$$

Corollary 1 below (a restatement of Theorem 2 in Berliant and Page (2001)) establishes the equivalence of the income tax-public goods mechanism design problem given by

$$
\max _{(y(\cdot), t(\cdot), z) \in \Psi \cap \Pi} \int_{W} u(w, y(w), t(y(w)), z) d \mu(w),
$$

and the menu design problem given by

$$
\max _{(C, z) \in R} \int_{W} \widehat{u}(w, C, z) d \mu(w) .
$$

Corollary 1 also describes how the optimal income tax function can be constructed from the optimal menu, public goods pair, and conversely, how the optimal menu, public goods pair can be constructed from the optimal income tax function. 
Corollary 1 (The Equivalence of the Mechanism Problem and the Menu Problem): Suppose [A-1]-[A-4] hold. Let $\mu$ be any finite measure equivalent to the measure $\eta$. Then the income tax-public goods mechanism design problem (20) has a solution if and only if the menu design problem (21) has a solution. In particular, the following statements are true:

(1) If the income tax-public goods mechanism $(y(\cdot), t(\cdot), z) \in \Psi \cap \Pi$

$$
\text { maximizes } \int_{W} u(w, y(w), t(y(w)), z) d \mu(w) \text { over } \Psi \cap \Pi
$$

then the pair $(\operatorname{cl}[G r(t(\cdot)], z)$, where $c l[G r(t(\cdot)]$ is the closure of the graph of the income tax function $t(\cdot)$ and $z$ is the public goods vector in $\mathbb{R}_{+}^{k}$, is contained in $R$ and

$$
\text { maximizes } \int_{W} \widehat{u}(w, C, z) d \mu(w) \text { over } R \text {. }
$$

(2) If the menu-public goods pair $(C, z) \in R$

$$
\text { maximizes } \int_{W} \widehat{u}(w, C, z) d \mu(w) \text { over } R
$$

then the mechanism $(y(\cdot), t(\cdot), z)$ constructed in (a) and (b) below is contained in $\Psi \cap \Pi$ and

$$
\text { maximizes } \int_{W} u(w, y(w), t(y(w)), z) d \mu(w) \text { over } \Psi \cap \Pi \text {. }
$$

(a) $y(\cdot)$ is the direct income function and $z$ the vector of public goods corresponding to a direct income tax-public goods mechanism

$$
(y(\cdot), \tau(\cdot), z) \in M(W, \mathbb{Y}) \times M(W, \mathbb{T}) \times \mathbb{R}_{+}^{k}
$$

such that $(y(w), \tau(w)) \in \Phi(w, C, z)$ for all $w \in W$ and

$$
\int_{W}\left(\tau(w)-\frac{h(z)}{\eta(W)}\right) d \eta(w) \geq 0 ;
$$

(b) $t(\cdot): \mathbb{Y} \rightarrow \mathbb{T}$ is the $(B(\mathbb{Y}), B(\mathbb{T}))$-measurable function given by

$$
t(y):=\min \{\tau:(y, \tau) \in C\} \text { for all } y \in \mathbb{Y} \text {. }
$$

Note that given $(C, z) \in \Lambda \times \mathbb{R}_{+}^{k}$, we have for any direct mechanism $(y(\cdot), \tau(\cdot)) \in$ $\Sigma(C, z)$

$$
u(w, y(w), \tau(w), z)=\widehat{u}(w, C, z) \text { for all } w \in W
$$


Moreover, for the unique income tax function, $t(\cdot): \mathbb{Y} \rightarrow \mathbb{T}$ constructed from menu $C \in \Lambda$ in accordance with part 2(b) of Corollary 1 , we have for any direct mechanism any direct mechanism $(y(\cdot), \tau(\cdot)) \in \Sigma(C, z)$

$$
(y(w), t(y(w)))=(y(w), \tau(w)) \text { for all } w \in W .
$$

Because the objective function,

$$
(C, z) \rightarrow \int_{W} \widehat{u}(w, C, z) d \mu(w)
$$

in the menu design problem is continuous, the compactness of the constraint set $R$ implies that the menu design problem has a solution (see Theorem 3 in Berliant and Page (2001)), and thus implies via Corollary 1 that the original income tax-public goods mechanism design problem ((6)-(9)) has a solution (see Theorem 1 in Berliant and Page (2001)).

\section{The Problem of Budget Balancedness}

\subsection{Tax Convexity and Aggregate Income and Tax Revenue}

The equivalence of the mechanism design problem and the menu design problem established in Corollary 1 allows us to approach the problem of budget balancedness via the simpler menu design problem. We begin by considering the best response mapping, $w \rightarrow \Phi(w, C, z)$, corresponding to the menu, public goods pair $(C, z)$. The closed set $\Phi(w, C, z)$ is the type $w$ agent's set of optimal income and tax liability choices given $(C, z)$. Since for all $w \in W$ and all $(C, z) \in \Lambda \times \mathbb{R}_{+}^{k}, \Phi(w, C, z) \subset K$, and since $K$ is a compact subset of $\mathbb{R}^{2}$, the collection of best response mappings,

$$
\left\{\Phi(\cdot, C, z):(C, z) \in \Lambda \times \mathbb{R}_{+}^{k}\right\}
$$

is $\eta$-integrably bounded. ${ }^{13}$

Now consider the set-valued mapping

$$
(C, z) \rightarrow \int_{W} \Phi(w, C, z) d \eta(w)
$$

\footnotetext{
${ }^{13}$ Thus, there exists a $\eta$-integrable function

$$
g(\cdot): W \rightarrow \mathbb{R}
$$

such that for any menu public goods pair $(C, z) \in \Lambda \times \mathbb{R}_{+}^{k}$,

$$
\|(y, \tau)\| \leq g(w)
$$

for all $w \in W$ and $(y, \tau) \in \Phi(w, C, z)$.
} 
where

$$
\int_{W} \Phi(w, C, z) d \eta(w):=\left\{\int_{W} f(w) d \eta(w): f(\cdot)=(y(\cdot), \tau(\cdot)) \in \Sigma(C, z)\right\} .
$$

The set $\int_{W} \Phi(w, C, z) d \eta(w)$ is the integral of the best response mapping $\Phi(\cdot, C, z)$. Moreover, $\int_{W} \Phi(w, C, z) d \eta(w)$ is the set we have referred to in Subsection 3.3.1 as the tax and income possibilities set (TIPS). Our next proposition summarizes the properties of this set, as well as how this set varies with changes in the menu and public goods pair.

Proposition 4 (1) For each $(C, z) \in \Lambda \times \mathbb{R}_{+}^{k}, \int_{W} \Phi(w, C, z) d \eta(w)$ is a nonempty, compact subset of $\mathbb{R}^{2}$. Moreover, if the measure space of agent types $(W, \Sigma, \eta)$ is atomless, then

$$
\int_{W} \Phi(w, C, z) d \eta(w) \text { is convex. }
$$

(2) The mapping $(C, z) \rightarrow \int_{W} \Phi(w, C, z) d \eta(w)$ is upper semicontinuous on $\Lambda \times \mathbb{R}_{+}^{k}$.

The integral of the best response mapping $w \rightarrow \Phi(w, C, z)$,

$$
\int_{W} \Phi(w, C, z) d \eta(w)
$$

contains all aggregate income and tax revenue pairs, $(Y, T)$, attainable under menu public goods pair $(C, z) \in \Lambda \times \mathbb{R}_{+}^{k}$ via some incentive compatible direct mechanism

$$
(y(\cdot), \tau(\cdot)) \in \Sigma(C, z) .
$$

In particular,

$$
\begin{aligned}
& \text { if }(Y, T) \in \int_{W} \Phi(w, C, z) d \eta(w) \text {, then there exists } \\
& (y(\cdot), \tau(\cdot)) \in \Sigma(C, z) \text { such that } \\
& Y=\int_{W} y(w) d \eta(w) \text { and } T=\int_{W} \tau(w) d \eta(w) .
\end{aligned}
$$

Moreover, for the income tax function, $t(\cdot): \mathbb{Y} \rightarrow \mathbb{T}$, uniquely determined by menu $C \in \Lambda$ via the construction described in part 2(b) of Corollary 1 , we have

$$
(y(w), \tau(w))=(y(w), t(y(w))) \text { for all } w \in W
$$

and thus,

$$
T=\int_{W} t(y(w)) d \eta(w) .
$$


Definition 5 (Tax Convexity): We say that the tax and income possibilities set,

$$
\int_{W} \Phi(w, C, z) d \eta(w)
$$

is tax convex if for all $\left(Y^{1}, T^{1}\right)$ and $\left(Y^{2}, T^{2}\right)$ contained in $\int_{W} \Phi(w, C, z) d \eta(w)$ and for all

$$
T_{\lambda}:=(1-\lambda) T^{1}+\lambda T^{2}, \lambda \in[0,1],
$$

there exists $Y_{\lambda}$ such that

$$
\left(Y_{\lambda}, T_{\lambda}\right) \in \int_{W} \Phi(w, C, z) d \eta(w)
$$

If $\int_{W} \Phi(w, C, z) d \eta(w)$ is convex, then it is automatically tax convex. However, as illustrated in Figure 4 above, the converse is not true.

\subsection{Tax Convexity and Budget Balancedness}

Our next result on budget balancedness states that if the tax and income possibilities set, $\int_{W} \Phi\left(w, C^{*}, z^{*}\right) d \eta(w)$, is tax convex at the optimal menu public goods pair $\left(C^{*}, z^{*}\right) \in \Lambda \times \mathbb{R}_{+}^{k}$, then there exists an optimal income tax-public goods mechanism that is budget balancing.

Theorem 4 (Tax Convexity and Budget Balancedness):

Suppose $[A-1]-[A-4]$ hold and let $\left(C^{*}, z^{*}\right) \in R$ be a solution to the menu design problem given by

$$
\max _{(C, z) \in R} \int_{W} \widehat{u}(w, C, z) d \mu(w) .
$$

If $\int_{W} \Phi\left(w, C^{*}, z^{*}\right) d \eta(w)$ is tax convex, then there exists an optimal income tax-public goods mechanism,

$$
\left(y^{*}(\cdot), t^{*}(\cdot), z^{*}\right) \in \Psi \cap \Pi,
$$

corresponding to $\left(C^{*}, z^{*}\right) \in R$ that is budget balancing. In particular, there exists a income tax-public goods mechanism $\left(y^{*}(\cdot), t^{*}(\cdot), z^{*}\right) \in \Psi \cap \Pi$,

$$
\text { maximizing } \int_{W} u(w, y(w), t(y(w)), z) d \mu(w) \text { over } \Psi \cap \Pi
$$

such that

$$
\int_{W} t^{*}\left(y^{*}(w)\right) d \eta(w)=h\left(z^{*}\right)
$$


By Theorem 3 in Berliant and Page (2001), the menu design problem has a solution, say $\left(C^{*}, z^{*}\right) \in R$. By Theorem 4 , tax convexity of the tax and income possibilities set at the optimal menu-public goods pair $\left(C^{*}, z^{*}\right) \in R$ is sufficient to guarantee the existence of an optimal income tax-public goods mechanism $\left(y^{*}(\cdot), t^{*}(\cdot), z^{*}\right) \in$ $\Psi \cap \Pi$ satisfying budget balancedness. We now identify two conditions, capacity constrained single crossing and the atomless condition, and show that these conditions guarantee tax convexity.

\subsection{Two Conditions Sufficient for Tax Convexity}

\subsubsection{The Capacity Constrained Single Crossing Condition}

Capacity constrained single crossing essentially ensures tax convexity, but the connection between single crossing and tax convexity is subtle.

Theorem 5 (Capacity Constrained Single Crossing and Tax Convexity):

Suppose [A-1]-[A-4] hold. Moreover, suppose that the set of agent types $W$ is a subset of the real numbers equipped with the usual ordering and that capacity constrained single crossing holds, so that [A-5] and [A-6] hold. Then given any menu public goods pair $(C, z) \in \Lambda \times \mathbb{R}_{+}^{k}$, there exists another menu $D \in \Lambda$ such that

$$
\begin{gathered}
\text { (1) } \widehat{u}(w, C, z)=\widehat{u}(w, D, z) \text { for all } w \in W, \\
\text { (2) } \int_{W} \Phi(w, D, z) d \eta(w) \text { is tax convex, } \\
\text { (3) } \int_{W} \Phi(w, C, z) d \eta(w) \subseteq \int_{W} \Phi(w, D, z) d \eta(w) \text {. }
\end{gathered}
$$

Let $\left(C^{*}, z^{*}\right) \in R$ be the solution to the menu design problem (21) guaranteed by Theorem 3 in Berliant and Page (2001). Under [A-5] and [A-6], it follows from Theorem 5 that there exists another menu $D^{*} \in \Lambda$ such that $\left(D^{*}, z^{*}\right) \in R$ also solves the menu design problem (21), and more importantly, such that $\int_{W} \Phi\left(w, D^{*}, z^{*}\right) d \eta(w)$ is tax convex. The existence of an optimal, budget balancing income tax and public goods mechanism, $\left(y^{*}(\cdot), t^{*}(\cdot), z^{*}\right) \in \Psi \cap \Pi$, corresponding to $\left(D^{*}, z^{*}\right) \in R$ then follows from Theorem 4. Thus, the proof of Theorem 1 follows from Theorem 3 in Berliant and Page (2001) and Theorems 4 and 5 above.

\subsubsection{The Atomless Condition}

The atomless condition also ensures tax convexity. The connection between atomlessness and tax convexity is quite direct. 
Theorem 6 Suppose [A-1]-[A-4] hold. Moreover, suppose that the measure space of agent types $(W, \Sigma, \eta)$ is atomless, so that $[A-7]$ holds. Then given any menu public goods pair $(C, z) \in \Lambda \times \mathbb{R}_{+}^{k}$,

$$
\int_{W} \Phi(w, C, z) d \eta(w) \text { is tax convex. }
$$

The proof of Theorem 6 follows immediately from part (1) of Proposition 4 which states that

if the measure space of agent types $(W, \Sigma, \eta)$ is atomless, then

$$
\int_{W} \Phi(w, C, z) d \eta(w) \text { is convex for all }(C, z) \in \Lambda \times \mathbb{R}_{+}^{k} .
$$

In many applications, the atomless condition will be satisfied automatically (see Example 4).

Let $\left(C^{*}, z^{*}\right) \in R$ be the solution to the menu design problem (21) guaranteed by Theorem 3 in Berliant and Page (2001). Under [A-7] it follows from Theorem 6 that $\int_{W} \Phi\left(w, C^{*}, z^{*}\right) d \eta(w)$ is tax convex. The existence of an optimal, budget balancing income tax and public goods mechanism, $\left(y^{*}(\cdot), t^{*}(\cdot), z^{*}\right) \in \Psi \cap \Pi$, corresponding to $\left(C^{*}, z^{*}\right) \in R$ then follows from Theorem 4 . Thus, the proof of Theorem 2 follows from Theorem 3 in Berliant and Page (2001) and Theorems 4 and 6 above.

\section{Proofs}

\subsection{Proof of Proposition 3}

If $R=\emptyset$, then the proposition is true. Suppose $R \neq \emptyset$. First, note that by assumption [A-2](2) and the compactness of $P_{f}(K), R$ is bounded. The proof will be complete if we can show that $R$ is closed. Consider the net tax contribution function $(w, C, z) \rightarrow$ $\sigma(w, C, z)$ given by

$$
(w, C, z) \rightarrow \sigma(w, C, z):=\max \left\{\tau-\frac{h(z)}{\eta(W)}:(y, \tau) \in \Phi(w, C, z)\right\} .
$$

Here, $\frac{h(z)}{\eta(W)}$ is the per capita cost of providing public goods $z$. The quantity $\sigma(w, C, z)$ is the maximum amount of net per capita tax contribution obtainable from a type $w$ agent consistent with incentive compatibility. Since $\Phi(w, C, z) \subset K$ is nonempty and compact, $\sigma(w, C, z)$ is well-defined for each $(w, C, z) \in W \times \Lambda \times \mathbb{R}_{+}^{k}$. Moreover, by Proposition 4.3 in Page (1992), $\sigma(\cdot, \cdot, \cdot)$ is $\Sigma \times B(\Lambda) \times B\left(\mathbb{R}_{+}^{k}\right)$-measurable and for each $w \in W, \sigma(w, \cdot, \cdot)$ is upper semicontinuous on $\Lambda \times \mathbb{R}_{+}^{k}$.

Now consider the real-valued function $\Delta(\cdot, \cdot)$, defined on $\Lambda \times \mathbb{R}_{+}^{k}$, given by

$$
\Delta(C, z):=\int_{W} \sigma(w, C, z) d \eta(w) .
$$


Since $\sigma(w, \cdot, \cdot)$ is upper semicontinuous on $\Lambda \times \mathbb{R}_{+}^{k}$, it follows from Fatou's Lemma (see Dudley $(1989)$ ) that $\Delta(\cdot, \cdot)$ is upper semicontinuous on $\Lambda \times \mathbb{R}_{+}^{k}$. Moreover, since

$$
R=\left\{(C, z) \in \Lambda \times \mathbb{R}_{+}^{k}: \Delta(C, z) \geq 0\right\}
$$

the closedness of $R$ follows from the definition of upper semicontinuity.

\subsection{Proof of Theorem 3}

PART (1): Let $(y(\cdot), t(\cdot), z) \in \Psi \cap \Pi$ and define menu $C$ as follows:

$$
C=c l[G r(t(\cdot))]
$$

where $c l$ denotes closure and $G r(t(\cdot))$ is the graph of the income tax function $t(\cdot)$. Thus,

$$
G r(t(\cdot))=\{(y, \tau) \in \mathbb{Y} \times \mathbb{T}: \tau=t(y)\} .
$$

First, note that since $t(\cdot)$ is defined on all of $\mathbb{Y}$,

$$
\operatorname{proj}_{\mathbb{Y}}[\operatorname{cl}[G r(t(\cdot))]]=\mathbb{Y} .
$$

Note also that since $s \leq t(y) \leq y$ for all $y \in \mathbb{Y}, s \leq t(y) \leq y$ for all $(y, \tau) \in \operatorname{cl}[G r(t(\cdot))]$. Thus, $c l[G r(t(\cdot))] \in \Lambda$. Finally, note that since $y(w) \in Y(w)$ for all $w \in W$,

$$
(y(w), t(y(w))) \in \operatorname{cl}[G r(t(\cdot))] \cap K(w) \text { for all } w \in W .
$$

Thus, for all $w \in W$,

$$
u(w, y(w), t(y(w)), z) \leq \max _{(y, \tau) \in d[\operatorname{Gr}(t(\cdot))] \cap K(w)} u(w, y, \tau, z)
$$

Suppose now that for some agent type $w^{\prime} \in W$ there is an income and tax liability pair $\left(y^{\prime}, \tau^{\prime}\right) \in \operatorname{cl}[G r(t(\cdot))] \cap K\left(w^{\prime}\right)$ such that

$$
u\left(w^{\prime}, y\left(w^{\prime}\right), t\left(y\left(w^{\prime}\right)\right), z\right)<u\left(w^{\prime}, y^{\prime}, \tau^{\prime}, z\right)
$$

By the essentiality of leisure $[\mathrm{A}-4]$, the pair $\left(y^{\prime}, \tau^{\prime}\right)$ is such that $y^{\prime}<m\left(w^{\prime}\right)$. Since $\left(y^{\prime}, \tau^{\prime}\right)$ is in the closure of the graph of $t(\cdot)$ and since $u\left(w^{\prime}, \cdot, \cdot, z\right)$ is continuous on $K\left(w^{\prime}\right)$, there is an income and tax liability pair $(\bar{y}, \bar{\tau}) \in K\left(w^{\prime}\right)$ with $\bar{y}<m\left(w^{\prime}\right)$ contained in the graph of $t(\cdot)$ such that

$$
u\left(w^{\prime}, y\left(w^{\prime}\right), t\left(y\left(w^{\prime}\right)\right), z\right)<u\left(w^{\prime}, \bar{y}, \bar{\tau}, z\right)
$$

Thus,

$$
u\left(w^{\prime}, y\left(w^{\prime}\right), t\left(y\left(w^{\prime}\right)\right), z\right)<u\left(w^{\prime}, \bar{y}, t(\bar{y}), z\right)
$$


where $\bar{\tau}=t(\bar{y})$. This contradicts the assumption that $(y(\cdot), t(\cdot), z) \in \Psi$ (i.e., the assumption that $(y(\cdot), t(\cdot), z)$ is incentive compatible). We must conclude therefore that for all $w \in W$,

$$
\begin{gathered}
(y(w), t(y(w))) \in \operatorname{cl}[G r(t(\cdot))] \cap K(w) \\
\text { and } \\
u(w, y(w), t(y(w)), z)=\max _{(y, \tau) \in c l[G r(t(\cdot))] \cap K(w)} u(w, y, \tau, z) .
\end{gathered}
$$

Thus, for $C=\operatorname{cl}[G r(t(\cdot))]$, we have

$$
(y(w), t(y(w))) \in \Phi(w, C, z) \text { for all } w \in W .
$$

Moreover, since $(y(\cdot), t(y(\cdot)))$ is a measurable selection from $\Phi(\cdot, C, z)$ (i.e., since $(y(\cdot), t(y(\cdot))) \in \Sigma(C, z))$, and since

$$
\int_{W}(t(y(w))-h(z)) d \eta(w) \geq 0
$$

we can conclude that $(C, z)=(\operatorname{cl}[G r(t(\cdot))], z) \in R$.

PART (2): Let $(C, z) \in R$ and let $(y(\cdot), \tau(\cdot)) \in \Sigma(C, z)$ be such that

$$
\int_{W}(\tau(w)-h(z)) d \eta(w) \geq 0
$$

Thus, the direct tax function $w \rightarrow \tau(w)$ finances public goods $z$ and

$$
u(w, y(w), \tau(w), z)=\max _{(y, \tau) \in C \cap K(w)} u(w, y, \tau, z) \text { for all } w \in W .
$$

Consider the income tax function $t(\cdot): \mathbb{Y} \rightarrow \mathbb{T}$ given by

$$
y \rightarrow t(y):=\min \{\tau \in \mathbb{T}:(y, \tau) \in C\} .
$$

Since $C$ is closed, $t(\cdot)$ is lower semicontinuous, and therefore $t(\cdot)$ is $(B(\mathbb{Y}), B(\mathbb{T}))$ measurable. Moreover, since

$$
\begin{aligned}
& (y, t(y)) \in C \text { for all } y \in \mathbb{Y}, \\
& s \leq t(y) \leq y \text { for all } y \in \mathbb{Y} .
\end{aligned}
$$

Claim 1:

$$
(y(w), \tau(w), z)=(y(w), t(y(w)), z) \text { for all } w \in W .
$$

If not, then for some agent type $w^{\prime} \in W, \tau\left(w^{\prime}\right) \neq t\left(y\left(w^{\prime}\right)\right)$. Since for all $w \in$ $W, t(y(w)):=\min \{\tau \in \mathbb{T}:(y(w), \tau) \in C\}, \tau\left(w^{\prime}\right) \neq t\left(y\left(w^{\prime}\right)\right)$ implies that $\tau\left(w^{\prime}\right)>$ $t\left(y\left(w^{\prime}\right)\right)$. But given monotonicity $[\mathrm{A}-3](4), \tau\left(w^{\prime}\right)>t\left(y\left(w^{\prime}\right)\right)$ contradicts the fact that

$$
u(w, y(w), \tau(w), z)=\max _{(y, \tau) \in C \cap K(w)} u(w, y, \tau, z) \text { for all } w \in W .
$$


Thus,

$$
\tau(w)=t(y(w)) \text { for all } w \in W
$$

and thus,

$$
\int_{W}(t(y(w))-h(z)) d \eta(w) \geq 0 .
$$

Claim 2: For all $w \in W$

$$
u(w, y(w), t(y(w)), z) \geq u(w, y, t(y), z) \text { for all } y \in Y(w) .
$$

Suppose not. Then for some agent type $w^{\prime} \in W$ and income $y^{\prime \prime} \in Y\left(w^{\prime}\right)$,

$$
u\left(w^{\prime}, y^{\prime \prime}, t\left(y^{\prime \prime}\right), z\right)>u\left(w^{\prime}, y\left(w^{\prime}\right), t\left(y\left(w^{\prime}\right)\right), z\right)=u\left(w^{\prime}, y\left(w^{\prime}\right), \tau\left(w^{\prime}\right), z\right) .
$$

Since $\left(y^{\prime \prime}, t\left(y^{\prime \prime}\right)\right) \in C \cap K(w),\left(^{*}\right)$ contradicts the fact that

$$
u(w, y(w), \tau(w), z)=\max _{(y, \tau) \in C \cap K(w)} u(w, y, \tau, z) \text { for all } w \in W .
$$

Thus,

$$
\begin{gathered}
(y(\cdot), t(\cdot), z) \in \Psi \cap \Pi, \\
\quad \text { and } \\
(y(w), t(y(w))) \in \Phi(w, C, z) \text { for all } w \in W .
\end{gathered}
$$

\subsection{Proof of Proposition 4}

PART (1): First recall that the set of measurable selectors

$\Sigma(C, z):=\{(y(\cdot), \tau(\cdot)) \in M(W, \mathbb{Y}) \times M(W, \mathbb{T}):(y(w), \tau(w)) \in \Phi(w, C, z) \forall w \in W\}$

is nonempty. Thus since the collection of best response mappings,

$$
\left\{\Phi(\cdot, C, z):(C, z) \in \Lambda \times \mathbb{R}_{+}^{k}\right\},
$$

is $\eta$-integrably bounded, it is easy to see that $\int_{W} \Phi(w, C, z) d \eta(w)$ is nonempty and bounded. To show that $\int_{W} \Phi(w, C, z) d \eta(w)$ is closed, consider a sequence $\left\{\left(Y_{n}, T_{n}\right)\right\}_{n}$ contained in $\int_{W} \Phi(w, C, z) d \eta(w)$ converging to $(Y, T) \in \mathbb{R}^{2}$, and let $\left\{\left(y_{n}(\cdot), \tau_{n}(\cdot)\right)\right\}_{n} \subset$ $\Sigma(C, z)$ be a corresponding sequence of measurable selectors such that for all $n$,

$$
\left(Y_{n}, T_{n}\right)=\left(\int_{W} y_{n}(w) d \eta(w), \int_{W} \tau_{n}(w) d \eta(w)\right) .
$$

Thus,

$$
\left(\lim _{n} Y_{n}, \lim _{n} T_{n}\right)=\left(\lim _{n} \int_{W} y_{n}(w) d \eta(w), \lim _{n} \int_{W} \tau_{n}(w) d \eta(w)\right) .
$$


Now consider the set-valued mapping,

$$
w \rightarrow \operatorname{Ls}\left\{\left(y_{n}(w), \tau_{n}(w)\right\},\right.
$$

where for each $w \in W$, the set $L s\left\{\left(y_{n}(w), \tau_{n}(w)\right\}\right.$ is the set of all limit points of the sequence $\left\{\left(y_{n}(w), \tau_{n}(w)\right)\right\}_{n}$. By Fatou's Lemma in several dimensions (e.g., see Artstein (1979)), there exists a $\Sigma$-measurable function, $\left(y^{f}(\cdot), \tau^{f}(\cdot)\right)$, and a set $N \in \Sigma$, with $\eta(N)=0$, such that

$$
\left(y^{f}(w), \tau^{f}(w)\right) \in L s\left\{\left(y_{n}(w), \tau_{n}(w)\right\} \text { for all } w \in W \backslash N,\right.
$$

and such that

$$
(Y, T)=\left(\int_{W} y^{f}(w) d \eta(w), \int_{W} \tau^{f}(w) d \eta(w)\right) .
$$

Let $\left(y^{\eta}(\cdot), \tau^{\eta}(\cdot)\right)$ be a measurable selection from the mapping $w \rightarrow L s\left\{\left(y_{n}(w), \tau_{n}(w)\right\}\right.$. Since this mapping is $\Sigma$-measurable with nonempty closed values, such a selection exists by the Kuratowski-Ryll-Nardzewski Selection Theorem (see Aliprantis and Border (1999), p. 567). Now define

$$
\begin{gathered}
y(w):=y^{f}(w) I_{W \backslash N}(w)+y^{\eta}(w) I_{N}(w), \\
\quad \text { and } \\
\tau(w):=\tau^{f}(w) I_{W \backslash N}(w)+\tau^{\eta}(w) I_{N}(w),
\end{gathered}
$$

where $I_{W \backslash N}(\cdot)$ and $I_{N}(\cdot)$ are the indicator functions for the sets $W \backslash N$ and $N$ respectively. We have $(y(w), \tau(w)) \in L s\left\{\left(y_{n}(w), \tau_{n}(w)\right\}\right.$ for all $w \in W$, and

$$
(Y, T)=\left(\int_{W} y(w) d \eta(w), \int_{W} \tau(w) d \eta(w)\right) .
$$

Moreover, since $\Phi(\cdot, C, z)$ is closed-valued

$$
L s\left\{\left(y_{n}(w), \tau_{n}(w)\right\} \subseteq \Phi(w, C, z) \text { for all } w \in W .\right.
$$

Thus, $(y(\cdot), \tau(\cdot)) \in \Sigma(C, z)$ and thus

$$
(Y, T)=\left(\int_{W} y(w) d \eta(w), \int_{W} \tau(w) d \eta(w)\right) \in \int_{W} \Phi(w, C, z) d \eta(w) .
$$

The convexity of $\int_{W} \Phi(w, C, z) d \eta(w)$ whenever $(W, \Sigma, \eta)$ is atomless follows directly from a classical result due to Richter (see Hildenbrand (1974), Theorem 3, page 62).

PART (2): Let $\left\{\left(C_{n}, z_{n}\right)\right\}_{n}$ be a sequence in $\Lambda \times \mathbb{R}_{+}^{k}$ converging to $(C, z) \in \Lambda \times \mathbb{R}_{+}^{k}$. Also, let $\left\{\left(Y_{n}, T_{n}\right)\right\}_{n}$ be a sequence in $\mathbb{R}^{2}$ such that for each $n$

$$
\left(Y_{n}, T_{n}\right) \in \int_{W} \Phi\left(w, C_{n}, z_{n}\right) d \eta(w) .
$$


Corresponding to the sequence $\left\{\left(Y_{n}, T_{n}\right)\right\}_{n}$ let $\left\{\left(y_{n}(\cdot), \tau_{n}(\cdot)\right)\right\}_{n}$ be a sequence of measurable functions such that for each $n,\left(y_{n}(\cdot), \tau_{n}(\cdot)\right) \in \Sigma\left(C_{n}, z_{n}\right)$ and

$$
\left(Y_{n}, T_{n}\right)=\left(\int_{W} y_{n}(w) d \eta(w), \int_{W} \tau_{n}(w) d \eta(w)\right) .
$$

Since $\left\{\left(Y_{n}, T_{n}\right)\right\}_{n}$ is bounded we can assume without loss of generality that $\left\{\left(Y_{n}, T_{n}\right)\right\}_{n}$ converges to some $(Y, T) \in \mathbb{R}^{2}$. Thus,

$$
(Y, T)=\left(\lim _{n} Y_{n}, \lim _{n} T_{n}\right)=\left(\lim _{n} \int_{W} y_{n}(w) d \eta(w), \lim _{n} \int_{W} \tau_{n}(w) d \eta(w)\right) .
$$

By Fatou's Lemma in several dimensions and the construction in Part 1 above, there exists a measurable selection, $(y(\cdot), \tau(\cdot))$, from the mapping

$$
w \rightarrow \operatorname{Ls}\left\{\left(y_{n}(w), \tau_{n}(w)\right\}\right.
$$

such that

$$
(Y, T)=\left(\int_{W} y(w) d \eta(w), \int_{W} \tau(w) d \eta(w)\right) .
$$

Moreover, since $\left\{\left(C_{n_{k}}, z_{n_{k}}\right)\right\}_{k}$ converges to $(C, z)$ and since for each $w \in W, \Phi(w, \cdot, \cdot)$ is upper semicontinuous on $\Lambda \times \mathbb{R}_{+}^{k}$,

$$
\begin{gathered}
\left(y_{n_{k}}(w), \tau_{n_{k}}(w)\right) \in \Phi\left(w, C_{n_{k}}, z_{n_{k}}\right) \text { for all } k \\
\text { and } \\
\left(\lim _{k} y_{n_{k}}(w), \lim _{k} \tau_{n_{k}}(w)\right)=(y(w), \tau(w)), \\
\text { imply that } \\
(y(w), \tau(w)) \in \Phi(w, C, z) .
\end{gathered}
$$

Thus, $(y(\cdot), \tau(\cdot)) \in \Sigma(C, z)$, and

$$
(Y, T)=\left(\int_{W} y(w) d \eta(w), \int_{W} \tau(w) d \eta(w)\right) \in \int_{W} \Phi(w, C, z) d \eta(w) .
$$

We conclude therefore that

$$
(C, z) \rightarrow \int_{W} \Phi(w, C, z) d \eta(w),
$$

is upper semicontinuous.

\subsection{Proof of Theorem 4}

Suppose that the direct mechanism,

$$
\left(y^{\prime}(\cdot), \tau^{\prime}(\cdot)\right) \in \Sigma\left(C^{*}, z^{*}\right)
$$


is such that

$$
\int_{W} \tau^{\prime}(w) d \eta(w)>h\left(z^{*}\right)
$$

Thus, the direct mechanism $\left(y^{\prime}(\cdot), \tau^{\prime}(\cdot)\right)$ generates excess revenue given public goods $z^{*}$. Let

$$
\left(Y^{\prime}, T^{\prime}\right)=\left(\int_{W} y^{\prime}(w) d \eta(w), \int_{W} \tau^{\prime}(w) d \eta(w)\right) .
$$

Now take menu $C^{*}$ and for each $n$ let the menu $C_{n}^{*}$ be defined as follows:

$$
\left(y_{n}, \tau_{n}\right) \in C_{n}^{*} \Leftrightarrow\left(y_{n}, \tau_{n}\right)=\left(y, s \vee \tau-\frac{1}{n}\right) \text { for some }(y, \tau) \in C^{*} .
$$

Here,

$$
s \vee \tau-\frac{1}{n}:=\max \left\{s, \tau-\frac{1}{n}\right\} .
$$

Observe that for some subset of agent types $E \in \Sigma$ of positive measure (i.e., with $\eta(E)>0$ ), we have $s<\tau^{\prime}(w)$ for $w \in E$. To see this note that if $s=\tau^{\prime}(w)$ a.e. $[\eta]$, this would contradict the fact that by $(23)$

$$
\int_{W} \tau^{\prime}(w) d \eta(w)>h\left(z^{*}\right) \geq s \eta(W) .
$$

Thus, we have for all $n$ and all $w \in E$

$$
s \vee \tau-\frac{1}{n}<\tau^{\prime}(w)
$$

Given assumption [A-3](4), monotonicity, we have for all $n$ and all measurable selectors $\left(y_{n}(\cdot), \tau_{n}(\cdot)\right)$ from $\Phi\left(\cdot, C_{n}^{*}, z^{*}\right)$ (i.e., for all $\left.\left(y_{n}(\cdot), \tau_{n}(\cdot)\right) \in \Sigma\left(C_{n}^{*}, z^{*}\right)\right)$,

$$
\begin{aligned}
& u\left(w, y_{n}(w), \tau_{n}(w), z^{*}\right) \\
& \geq u\left(w, y^{\prime}(w), s \vee \tau^{\prime}(w)-\frac{1}{n}, z^{*}\right) \\
& \geq u\left(w, y^{\prime}(w), \tau^{\prime}(w), z^{*}\right) \text { for all } w \in W
\end{aligned}
$$

and

$$
\begin{aligned}
& u\left(w, y_{n}(w), \tau_{n}(w), z^{*}\right) \\
& \geq u\left(w, y^{\prime}(w), s \vee \tau^{\prime}(w)-\frac{1}{n}, z^{*}\right) \\
& >u\left(w, y^{\prime}(w), \tau^{\prime}(w), z^{*}\right) \text { for all } w \in E .
\end{aligned}
$$

Thus, for all $n$ and all direct mechanisms $\left(y_{n}(\cdot), \tau_{n}(\cdot)\right) \in \Sigma\left(C_{n}^{*}, z^{*}\right)$ it must be true that

$$
\int_{W} \tau_{n}(w) d \eta(w)<h\left(z^{*}\right)
$$


In particular, if for some $n$

$$
\int_{W} \tau_{n}(w) d \eta(w) \geq h\left(z^{*}\right),
$$

then it follows that $\left(C_{n}^{*}, z^{*}\right) \in R$. Given inequalities (24) this would contradict the optimality of $\left(C^{*}, z^{*}\right) \in R$.

Observe now that $\left\{\left(C_{n}^{*}, z^{*}\right)\right\}_{n}$ converges to $\left(C^{*}, z^{*}\right)$ and let $\left\{\left(Y_{n}, T_{n}\right)\right\}_{n}$ be a sequence in $\mathbb{R}^{2}$ such that for each $n$

$$
\left(Y_{n}, T_{n}\right) \in \int_{W} \Phi\left(w, C_{n}^{*}, z^{*}\right) d \eta(w) .
$$

Since the collection of best response mappings, $\left\{\Phi(\cdot, C, z):(C, z) \in \Lambda \times \mathbb{R}_{+}^{k}\right\}$, is $\eta$ integrably bounded, we can assume without loss of generality that $\left\{\left(Y_{n}, T_{n}\right)\right\}_{n}$ converges to some $(Y, T) \in \mathbb{R}^{2}$. By the upper semicontinuity of the mapping

$$
(C, z) \rightarrow \int_{W} \Phi(w, C, z) d \eta(w)
$$

on $\Lambda \times \mathbb{R}_{+}^{k}$, we have

$$
(Y, T) \in \int_{W} \Phi\left(w, C^{*}, z^{*}\right) d \eta(w) .
$$

Let $(y(\cdot), \tau(\cdot)) \in \Sigma\left(C^{*}, z^{*}\right)$ be such that

$$
(Y, T)=\left(\int_{W} y(w) d \eta(w), \int_{W} \tau(w) d \eta(w)\right) .
$$

By inequality (25), we have

$$
\int_{W} \tau(w) d \eta(w) \leq h\left(z^{*}\right)
$$

Thus, we have

$$
\begin{gathered}
(Y, T)=\left(\int_{W} y(w) d \eta(w), \int_{W} \tau(w) d \eta(w)\right) \in \int_{W} \Phi\left(w, C^{*}, z^{*}\right) d \eta(w) \\
\text { with } T \leq h\left(z^{*}\right) \\
\quad \text { and } \\
\left(Y^{\prime}, T^{\prime}\right)=\left(\int_{W} y^{\prime}(w) d \eta(w), \int_{W} \tau^{\prime}(w) d \eta(w)\right) \in \int_{W} \Phi\left(w, C^{*}, z^{*}\right) d \eta(w) \\
\text { with } T^{\prime}>h\left(z^{*}\right) .
\end{gathered}
$$

Let $\lambda \in[0,1]$ be such that

$$
T_{\lambda}=(1-\lambda) T^{\prime}+\lambda T=h\left(z^{*}\right) .
$$


By the tax convexity of $\int_{W} \Phi\left(w, C^{*}, z^{*}\right) d \eta(w)$, there exists aggregate income $Y_{\lambda}$ generating $T_{\lambda}$ such that

$$
\left(Y_{\lambda}, T_{\lambda}\right) \in \int_{W} \Phi\left(w, C^{*}, z^{*}\right) d \eta(w)
$$

with corresponding measurable selector $\left(y^{*}(\cdot), \tau^{*}(\cdot)\right) \in \Sigma\left(C^{*}, z^{*}\right)$ such that

$$
\begin{gathered}
\left(Y_{\lambda}, T_{\lambda}\right)=\left(\int_{W} y^{*}(w) d \eta(w), \int_{W} \tau^{*}(w) d \eta(w)\right), \\
\text { and } \\
T_{\lambda}=\int_{W} \tau^{*}(w) d \eta(w)=h\left(z^{*}\right) .
\end{gathered}
$$

Let $t^{*}(\cdot): \mathbb{Y} \rightarrow \mathbb{T}$ be the income tax function uniquely determined by menu $C^{*}$. By part (2) of Corollary 1 the income tax-public goods mechanism $\left(y^{*}(\cdot), t^{*}(\cdot), z^{*}\right)$ constructed from mechanism $\left(y^{*}(\cdot), \tau^{*}(\cdot), z^{*}\right)$ and menu public goods pair $\left(C^{*}, z^{*}\right) \in R$ is contained in $\Psi \cap \Pi$ and

$$
\text { maximizes } \int_{W} u(w, y(w), t(y(w)), z) d \mu(w) \text { over } \Psi \cap \Pi \text {. }
$$

Moreover,

$$
\int_{W} \tau^{*}(w) d \eta(w)=\int_{W} t^{*}\left(y^{*}(w)\right) d \eta(w)=h\left(z^{*}\right)
$$

\subsection{Proof of Theorem 5}

(1) First, we will show that given $(C, z) \in \Lambda \times \mathbb{R}_{+}^{k}$, there exists $D \in \Lambda$ such that

$$
\widehat{u}(w, C, z)=\widehat{u}(w, D, z) \text { for all } w \in W .
$$

The proof will be divided into two parts:

(1) PART 1: Let $(C, z) \in R$ be given and let $\left(y^{u}(\cdot), \tau^{u}(\cdot)\right) \in \Sigma(C, z)$ be such that $y(w) \leq y^{u}(w)$ for all $w \in W$ and $(y(\cdot), \tau(\cdot)) \in \Sigma(C, z)$. Also, let $\left(y^{l}(\cdot), \tau^{l}(\cdot)\right) \in \Sigma(C, z)$ be such that $y^{l}(w) \leq y(w)$ for all $w \in W$ and $(y(\cdot), \tau(\cdot)) \in \Sigma(C, z)$. Call $\left(y^{u}(\cdot), \tau^{u}(\cdot)\right)$ and $\left(y^{l}(\cdot), \tau^{l}(\cdot)\right)$ the maximum and minimum income, incentive compatible direct mechanisms respectively. The direct income functions, $y^{l}(\cdot)$ and $y^{u}(\cdot)$, are given by

$$
\begin{gathered}
w \rightarrow y^{l}(w):=\min \left\{y: y \in \operatorname{proj}_{Y} \Phi(w, C, z)\right\}, \\
\text { and } \\
w \rightarrow y^{u}(w):=\max \left\{y: y \in \operatorname{proj}_{Y} \Phi(w, C, z)\right\},
\end{gathered}
$$

where $\operatorname{proj}_{Y} \Phi(w, C, z)$ is the projection of the best response set $\Phi(w, C, z)$ onto the $Y$-axis. Since $\operatorname{proj}_{Y} \Phi(\cdot, C, z)$ is compact-valued and measurable, the existence of the optimal measurable selectors, $y^{l}(\cdot)$ and $y^{u}(\cdot)$, is guaranteed by the Measurable Maximum Theorem (see Aliprantis and Border (1999), p. 570). The corresponding direct tax functions, $\tau^{l}(\cdot)$ and $\tau^{u}(\cdot)$, are then given by

$$
\begin{gathered}
w \rightarrow \tau^{l}(w):=\min \left\{\tau:\left(y^{l}(w), \tau\right) \in C\right\}, \\
\text { and } \\
w \rightarrow \tau^{u}(w):=\min \left\{\tau:\left(y^{u}(w), \tau\right) \in C\right\} .
\end{gathered}
$$


Next, for a given $(y(\cdot), \tau(\cdot)) \in \Sigma(C, z)$ define the function $w \rightarrow U(w, z)$ as follows:

$$
U(w, z):=u(w, y(w), \tau(w), z),
$$

and observe that

$$
U(w, z)=u\left(w, y^{\prime}(w), \tau^{\prime}(w), z\right) \text { for all }\left(y^{\prime}(\cdot), \tau^{\prime}(\cdot)\right) \in \Sigma(C, z) .
$$

Finally, define the following set-valued mapping: for all $w \in W$,

$$
\Gamma(w):=\left\{(y, \tau) \in K: y^{l}(w) \leq y \leq y^{u}(w) \text { and } u(w, y, \tau, z)=U(w, z)\right\} .
$$

For each agent type $w \in W, \Gamma(w)$ contains the income and tax liability combinations, with income levels between $y^{l}(w)$ and $y^{u}(w)$, lying on the type $w$ agent's indifference curve passing through $\left(y^{l}(w), \tau^{l}(w)\right)$ and $\left(y^{u}(w), \tau^{u}(w)\right)$. By the continuity and tax monotonicity of utility functions $([\mathrm{A}-3](1)$ and $[\mathrm{A}-3](4)) \Gamma(\cdot)$ takes nonempty, closed values. Moreover, the mapping $\Gamma(\cdot)$ is $\Sigma$-measurable (see Aliprantis and Border (1999), Chapter 17).

Our objective in this part of the proof is to show that capacity constrained single crossing implies that

$$
u(w, \Gamma(w), z) \geq u\left(w, \Gamma\left(w^{\prime}\right), z\right) \text { for all } w \text { and } w^{\prime} \text { in } W
$$

where

$$
u\left(w, \Gamma\left(w^{\prime}\right), z\right)=\max \left\{u(w, y, \tau, z):(y, \tau) \in \Gamma\left(w^{\prime}\right) \cap K(w)\right\} .
$$

Note that

$$
u(w, \Gamma(w), z)=u(w, y, \tau, z) \text { for all }(y, \tau) \in \Gamma(w)
$$

Let

$$
\Psi_{w}\left(w^{\prime}\right)=\left\{(y, \tau) \in \Gamma\left(w^{\prime}\right): u(w, y, \tau, z)=u\left(w, \Gamma\left(w^{\prime}\right), z\right)\right\} .
$$

Note also that for all $w$ and $w^{\prime}$ in $W, \Psi_{w}\left(w^{\prime}\right)$ is a nonempty closed subset of $\Gamma\left(w^{\prime}\right)$.

Suppose now that (26) fails. In particular, suppose that for some $w$ and $w^{\prime}$ in $W$,

$$
u\left(w, \Gamma\left(w^{\prime}\right), z\right)>u(w, \Gamma(w), z) .
$$

Inequality (29) implies that,

$$
\text { (i) }\left(y\left(w^{\prime}\right), \tau\left(w^{\prime}\right)\right) \notin \Psi_{w}\left(w^{\prime}\right) \text { for all }(y(\cdot), \tau(\cdot)) \in \Sigma(C, z) \text {. }
$$

Otherwise, if for some $(y(\cdot), \tau(\cdot)) \in \Sigma(C, z),\left(y\left(w^{\prime}\right), \tau\left(w^{\prime}\right)\right) \in \Psi_{w}\left(w^{\prime}\right)$, then inequality (29) and the definition of the mapping $\Gamma(\cdot)$ imply that

$$
u\left(w, y\left(w^{\prime}\right), \tau\left(w^{\prime}\right), z\right)>u(w, y(w), \tau(w), z),
$$


contradicting the fact that $(y(\cdot), \tau(\cdot)) \in \Sigma(C, z)$. Moreover, (29) together with assumption [A-4], essentiality of leisure, imply that

$$
\text { (ii) } y<m(w) \text { for all }(y, \tau) \in \Psi_{w}\left(w^{\prime}\right) \text {. }
$$

Let $\left(y^{\prime}, \tau^{\prime}\right) \in \Psi_{w}\left(w^{\prime}\right)$. Suppose $w^{\prime}>w$. By observations (ii) and (iii) above, $y^{l}\left(w^{\prime}\right)<$ $y^{\prime}<m(w)$. Thus, $\left(y^{l}\left(w^{\prime}\right), \tau^{l}\left(w^{\prime}\right)\right)$ is contained in $\Gamma\left(w^{\prime}\right) \cap K(w)$ but not contained in $\Psi_{w}\left(w^{\prime}\right)$. Thus, we have

$$
u\left(w, y^{\prime}, \tau^{\prime}, z\right)>u\left(w, y^{l}\left(w^{\prime}\right), \tau^{l}\left(w^{\prime}\right), z\right)
$$

By capacity constrained single crossing, [A-6],

$$
u\left(w^{\prime}, y^{\prime}, \tau^{\prime}, z\right)>u\left(w^{\prime}, y^{l}\left(w^{\prime}\right), \tau^{l}\left(w^{\prime}\right), z\right),
$$

contradicting the fact that $\left(y^{\prime}, \tau^{\prime}\right)$ and $\left(y^{l}\left(w^{\prime}\right), \tau^{l}\left(w^{\prime}\right)\right)$ are in $\Gamma\left(w^{\prime}\right)$.

Next, suppose $w^{\prime}<w$. By $[\mathrm{A}-6], m\left(w^{\prime}\right) \leq m(w)$ (i.e., $\left.K\left(w^{\prime}\right) \subseteq K(w)\right)$. Thus, we have $\Gamma\left(w^{\prime}\right) \cap K(w)=\Gamma_{z}\left(w^{\prime}\right)$ and by observation (i), $\Psi_{w}\left(w^{\prime}\right)$ is a proper subset of $\Gamma\left(w^{\prime}\right)$ with $y^{l}\left(w^{\prime}\right)<y^{\prime}<y^{u}\left(w^{\prime}\right)$ for all $\left(y^{\prime}, \tau^{\prime}\right) \in \Psi_{w}\left(w^{\prime}\right)$. We have

$$
u\left(w^{\prime}, y^{u}\left(w^{\prime}\right), \tau^{u}\left(w^{\prime}\right), z\right) \geq u\left(w^{\prime}, y^{\prime}, \tau^{\prime}, z\right) .
$$

In fact, $u\left(w^{\prime}, y^{u}\left(w^{\prime}\right), \tau^{u}\left(w^{\prime}\right), z\right)=u\left(w^{\prime}, y^{\prime}, \tau^{\prime}, z\right)$ because $\left(y^{u}\left(w^{\prime}\right), \tau^{u}\left(w^{\prime}\right)\right)$ and $\left(y^{\prime}, \tau^{\prime}\right)$ are in $\Gamma\left(w^{\prime}\right)$. By capacity constrained single crossing [A-6]

$$
u\left(w, y^{u}\left(w^{\prime}\right), \tau^{u}\left(w^{\prime}\right), z\right) \geq u\left(w, y^{\prime}, \tau^{\prime}, z\right),
$$

contradicting the fact that $\left(y^{\prime}, \tau^{\prime}\right) \in \Psi_{w}\left(w^{\prime}\right)$ and $\left(y^{u}\left(w^{\prime}\right), \tau^{u}\left(w^{\prime}\right)\right) \in \Gamma\left(w^{\prime}\right) \backslash \Psi_{w}\left(w^{\prime}\right)$ (see observation (ii) above).

(1) PART 2: Let

$$
C_{\Gamma}=c l\left(\cup_{w} \Gamma(w)\right) \text { and } D=C \cup C_{\Gamma}
$$

where $c l$ denotes closure. Also, let

$\Sigma(\Gamma(\cdot))$ denote the set of all $\Sigma$-measurable selections from the mapping $\Gamma(\cdot)$.

Our objective in this part of the proof is to show that

$$
\Sigma(D, z)=\Sigma(\Gamma(\cdot))
$$

Let $(y(\cdot), \tau(\cdot)) \in \Sigma(D, z)$, but suppose $(y(\cdot), \tau(\cdot)) \notin \Sigma(\Gamma(\cdot))$. Thus, for some $\bar{w} \in W,(y(\bar{w}), \tau(\bar{w})) \notin \Gamma(\bar{w})$. Let $(\bar{y}, \bar{\tau}) \in \Gamma(\bar{w})$. Since $(y(\cdot), \tau(\cdot)) \in \Sigma(D, z)$ and $\Gamma(\bar{w}) \subset D$, we have

$$
u(\bar{w}, y(\bar{w}), \tau(\bar{w}), z) \geq u(\bar{w}, \bar{y}, \bar{\tau}, z)
$$


and since $(y(\bar{w}), \tau(\bar{w})) \notin \Gamma(\bar{w})$, it must be true that

$$
u(\bar{w}, y(\bar{w}), \tau(\bar{w}), z)>u(\bar{w}, \bar{y}, \bar{\tau}, z) .
$$

If $(y(\bar{w}), \tau(\bar{w})) \in C_{\Gamma}$, then by continuity [A-3](1) and closure (i.e., the fact that $\left.C_{\Gamma}=c l\left(\cup_{w} \Gamma(w)\right)\right)$ there exists $\left(y^{\prime}, \tau^{\prime}\right) \in \cup_{w} \Gamma(w)$ such that

$$
u\left(\bar{w}, y^{\prime}, \tau^{\prime}, z\right)>u(\bar{w}, \bar{y}, \bar{\tau}, z)
$$

Let $\left(y^{\prime}, \tau^{\prime}\right) \in \Gamma\left(w^{\prime}\right)$ for some $w^{\prime} \neq \bar{w}$. Given that $u\left(\bar{w}, y^{\prime}, \tau^{\prime}, z\right)>u(\bar{w}, \bar{y}, \bar{\tau}, z)$, we have by the essentiality of leisure $[\mathrm{A}-4]$ that

$$
\left(y^{\prime}, \tau^{\prime}\right) \in \Gamma\left(w^{\prime}\right) \cap K(\bar{w}) .
$$

This implies that

$$
u\left(\bar{w}, \Gamma\left(w^{\prime}\right), z\right)>u(\bar{w}, \Gamma(\bar{w}), z),
$$

contradicting (26) established above.

If $(y(\bar{w}), \tau(\bar{w})) \in C$, then $(y(\bar{w}), \tau(\bar{w})) \in \Phi(\bar{w}, C, z)$. We have

$$
u\left(\bar{w}, y^{l}(\bar{w}), \tau^{l}(\bar{w}), z\right)=u(\bar{w}, y(\bar{w}), \tau(\bar{w}), z)>u(\bar{w}, \bar{y}, \bar{\tau}, z)=u\left(\bar{w}, y^{l}(\bar{w}), \tau^{l}(\bar{w}), z\right)
$$

a contradiction. Recall that $\left(y^{l}(\cdot), \tau^{l}(\cdot)\right) \in \Sigma(C, z)$ is the minimum income, incentive compatible direct mechanism in $\Sigma(C, z)$. Thus, we must conclude that

$$
(y(\bar{w}), \tau(\bar{w})) \in \Gamma(\bar{w})
$$

and therefore that

$$
\Sigma(D, z) \subseteq \Sigma(\Gamma(\cdot))
$$

Let $(y(\cdot), \tau(\cdot)) \in \Sigma(\Gamma(\cdot))$. In order to show that $(y(\cdot), \tau(\cdot)) \in \Sigma(D, z)$, we must show that for all $w \in W$

$$
u(w, y(w), \tau(w), z)=\max \{u(w, y, \tau, z):(y, \tau) \in D \cap K(w)\}:=\widehat{u}(w, D, z)
$$

We have for all $w \in W,(y(w), \tau(w)) \in D \cap K(w)$. Thus,

$$
u(w, y(w), \tau(w), z) \leq \max \{u(w, y, \tau, z):(y, \tau) \in D \cap K(w)\}
$$

Suppose that for some $\bar{w} \in W$

$$
u(\bar{w}, y(\bar{w}), \tau(\bar{w}), z)<\max \{u(\bar{w}, y, \tau, z):(y, \tau) \in D \cap K(\bar{w})\}
$$

Let $(\bar{y}, \bar{\tau}) \in D \cap K(\bar{w})$ be such that

$$
u(w, \bar{y}, \bar{\tau}, z)=\max \{u(w, y, \tau, z):(y, \tau) \in D \cap K(w)\}:=\widehat{u}(w, D, z) .
$$


Inequality (30), together with the definition of the mapping $\Gamma(\cdot)$, imply that $(\bar{y}, \bar{\tau}) \notin C$ and in particular that

$$
(\bar{y}, \bar{\tau}) \in\left(\left(C_{\Gamma} \cap K(w)\right) \backslash C\right) \subset D \cap K(\bar{w}) .
$$

Moreover, by [A-4], the essentiality of leisure, $\bar{y}<m(\bar{w})$. By continuity [A-3] (1) and closure (i.e., the fact that $\left.C_{\Gamma}=c l\left(\cup_{w} \Gamma(w)\right)\right)$ there exists $\left(y^{\prime}, \tau^{\prime}\right) \in \Gamma\left(w^{\prime}\right)$ for some $w^{\prime} \neq \bar{w}$ such that

$$
u(\bar{w}, y(\bar{w}), \tau(\bar{w}), z)<u\left(\bar{w}, y^{\prime}, \tau^{\prime}, z\right)
$$

But this implies that

$$
u(\bar{w}, \Gamma(\bar{w}), z)<u\left(\bar{w}, \Gamma\left(w^{\prime}\right), z\right),
$$

contradicting $(26)$ established above. Thus, $(y(\cdot), \tau(\cdot)) \in \Sigma(D, z)$ and we conclude that

$$
\Sigma(D, z)=\Sigma(\Gamma(\cdot))
$$

Given the definition of the mapping $w \rightarrow \Gamma(w)$ and the fact that $\Sigma(D, z)=\Sigma(\Gamma(\cdot))$, we conclude that

$$
\widehat{u}(w, C, z)=\widehat{u}(w, D, z) \text { for all } w \in W .
$$

(2) Next we show that

$$
\int_{W} \Phi(w, D, z) d \eta(w) \text { is tax convex. }
$$

Let $\left(Y^{1}, T^{1}\right)$ and $\left(Y^{2}, T^{2}\right)$ be in $\int_{W} \Phi(w, D, z) d \eta(w)$ and let

$$
T_{\lambda}=(1-\lambda) T^{1}+\lambda T^{2} .
$$

Also, let $\left(y^{1}(\cdot), \tau^{1}(\cdot)\right)$ and $\left(y^{2}(\cdot), \tau^{2}(\cdot)\right)$ in $\Sigma(D, z)$ be such that

$$
T^{1}=\int_{W} \tau^{1}(w) d \eta(w) \text { and } T^{2}=\int_{W} \tau^{2}(w) d \eta(w) .
$$

By the continuity of utility functions, [A-3](1), for each $w \in W$ the set $\Gamma(w)$ is tax convex; that is, the projection of $\Gamma(w)$ onto the tax liability axis (i.e., the $\tau$ axis) is a closed interval. Let

$$
\tau_{\lambda}(\cdot)=(1-\lambda) \tau^{1}(\cdot)+\lambda \tau^{2}(\cdot),
$$

and define the set-valued mapping $\Upsilon(\cdot)$ as follows:

$$
\Upsilon(w):=\left\{y \in\left[y^{l}(w), y^{u}(w)\right]: u\left(w, y, \tau_{\lambda}(w), z\right)=u\left(w, y^{1}(w), \tau^{1}(w), z\right)\right\} .
$$

By the tax convexity of $\Gamma(w)$ for each $w \in W, \Upsilon(w)$ is nonempty. Moreover, since the mapping $\Upsilon(\cdot)$ is $\Sigma$-measurable and closed-valued, it follows from the KuratowskiRyll-Nardzewski Selection Theorem (see Aliprantis and Border (1999), pages 563 and $567)$ that there exists an $\Sigma$-measurable function, $y_{\lambda}(\cdot)$, such that

$$
y_{\lambda}(w) \in \Upsilon(w) \text { for all } w \in W .
$$


Thus,

$$
\begin{gathered}
\left(y_{\lambda}(\cdot), \tau_{\lambda}(\cdot)\right) \in \Sigma(\Gamma(\cdot))=\Sigma(D, z) \\
\left(Y_{\lambda}, T_{\lambda}\right)=\left(\int_{W} y_{\lambda}(w) d \eta(w), \int_{W} \tau_{\lambda}(w) d \eta(w)\right) \in \int_{W} \Phi(w, D, z) d \eta(w) .
\end{gathered}
$$

(3) Finally, we show that

$$
\int_{W} \Phi(w, C, z) d \eta(w) \subseteq \int_{W} \Phi(w, D, z) d \eta(w) .
$$

But (31) is an immediate consequence of the fact that

$$
\Sigma(C, z) \subseteq \Sigma(\Gamma(\cdot))=\Sigma(D, z) .
$$

\section{References}

[1] Aliprantis, C. D. and Border, K. C. (1999) Infinite Dimensional Analysis: A Hitchhiker's Guide, Springer, Berlin.

[2] Artstein, Z. (1979) "A Note on Fatou's Lemma in Several Dimensions," Journal of Mathematical Economics 6, 277-282.

[3] Berge, C. (1963) Topological Spaces, Macmillan, New York.

[4] Berliant, M. and Page, F. H., Jr. (1996) "Incentives and Income Taxation: The Implementation of Individual Revenue Requirement Functions," Ricerche Economiche 50, 389-400.

[5] Berliant, M. and Page, F. H., Jr. (2001) "Income Taxes and the Provision of Public Goods: Existence of an Optimum," Econometrica 69, 771-784.

[6] Conley, J. P. and Wooders, M. H. (2001) "Tiebout Economies with Differential Genetic Types and Endogenously Chosen Crowding Characteristics," Journal of Economic Theory 98, 261-294.

[7] Diamond, P. A. and Mirrlees, J. (1971) "Optimal Taxation and Public Production," American Economic Review 61, 8-27, 261-278.

[8] Dudley, R. M. (1989) Real Analysis and Probability, Wadsworth\& Brooks-Cole, Pacific Grove.

[9] Hammond, P. J. (1979) "Straightforward Individual Incentive Compatibility in Large Economies," Review of Economic Studies 46, 263-282.

[10] Hildenbrand, W. (1974) Core and Equilibria of a Large Economy, Princeton University Press, Princeton. 
[11] Himmelberg, C. J. (1975) "Measurable Relations" Fundamenta Mathematicae LXXXVII, 53-72.

[12] Holmstrom, B. (1979) "Moral Hazard and Observability," Bell Journal of Economics 10, 74-91.

[13] Holmstrom, B. (1984) "On the Theory of Delegation," in: M. Boyer and R. Kihlstrom, eds., Bayesian Models in Economic Theory, North Holland, Amsterdam.

[14] Konishi, H. (1995) "A Pareto-Improving Commodity Tax Reform Under a Smooth Nonlinear Income Tax," Journal of Public Economics 56, 413-446.

[15] Mirrlees, J. (1971) "An Exploration in the Theory of Optimum Income Taxation," Review of Economic Studies 38, 175-208.

[16] Mirrlees, J. (1976) "The Optimal Structure of Incentives and Authority within an Organization," Bell Journal of Economics 7, 105-131.

[17] Myles, G. D. (1995) Public Economics, Cambridge University Press.

[18] Page, F. H., Jr. (1992) "Mechanism Design for General Screening Problems with Moral Hazard," Economic Theory 2, 265-281.

[19] Vickrey, W. (1945) "Measuring Marginal Utility by Reactions to Risk," Econometrica 13, 319-333. 